\title{
SOME PROPERTIES OF THE DOUBLE LAPLACE TRANSFORMATION
}

\author{
BY \\ GERALDINE A. COON AND DOROTHY L. BERNSTEIN
}

The double Laplace integral taken over the first quadrant $Q$

$$
\int_{Q} e^{-s x-t y} F(x, y) d(x, y), \quad x, y \text { real, } s, t \text { complex, }
$$

has been the subject of several systematic investigations $\left({ }^{1}\right)$. It is the purpose of this paper to develop further properties of the transformation including conditions for transforming derivatives, integrals, and convolutions. The fundamental assumption is bounded convergence of the integral, but several new types of convergence, less restrictive than absolute convergence, will be introduced for use in problems involving iteration of the transform.

1. Introduction. The Laplace transform is a nonabsolutely convergent infinite integral; it is therefore necessary to investigate the behavior of functions which are summable in every finite rectangle without necessarily being summable in the infinite quadrant. In this connection classes of functions called $\Sigma_{x}, \Sigma, \bar{\Sigma}$ are introduced. It is also useful to introduce classes $A_{x}, A, A^{*}$, generalizations of functions absolutely continuous in every finite interval or rectangle. This section contains properties of these classes which are needed in later proofs. It is possible to begin reading with $\$ 2$ where the double transform is first defined and to refer to the lemmas of this section when necessary.

Let $S$ be a measurable kernel of the interval $I: 0 \leqq x<\infty$, that is, $S \subseteq I$ and the Lebesgue measure $m(I-S)=0$. A function $F$ defined in $S$ is said to be in class $\Sigma_{x}$ if the equivalent function $F_{0}$ defined in $I$ by

$$
F_{0}(x)=F(x) \quad(x \in S) ; \quad F_{0}(x)=0 \quad(x \in I-S)
$$

is Lebesgue summable in every finite interval $I_{X}: 0 \leqq x \leqq X$. By $\int_{0}^{X} F(x) d x$ we mean $\int_{I_{X}} F_{0}(x) d x$, which is equal to $\int_{S \cdot I_{X}} F(x) d x$. The function $\Phi$ defined in $I$ by the equation $\phi(x)=\int_{0}^{x} F(u) d u$ is absolutely continuous in every $I_{X}$.

A function $F(x)$ defined on $I^{0}: 0<x<\infty$ is said to be of class $A_{x}$ whenever

Received by the editors December 20, 1951 .

(1) D. L. Bernstein, The double Laplace integral, Dissertation, Brown University, 1939.

L. Amerio, Sulla trasformata doppia di Laplace, Atti della Reale Accademia d'Italia. Memorie della Classe di Scienze Fisiche, Matematiche e Naturali vol. 7 (1941) pp. 707-780.

D. L. Bernstein, The double Laplace integral, Duke Math. J. vol. 8 (1941) pp. 460-496. This paper considers the Stieltjes integral $\int_{Q} e^{-s x-t y} d \phi(x, y)$; the transform we consider is a special case of this when $\phi(x, y)=\int_{R_{X Y}} F(x, y) d(x, y)$. 
$F(+0)$ exists and is finite and the function $F_{1}$ defined on $I$ by the equations $F_{1}(0)=F(+0), F_{1}(x)=F(x)$ for $x>0$ is absolutely continuous on every $I_{X}$. A necessary and sufficient condition that $F(x)$, defined on $I^{0}$, be in class $A_{x}$ is that $F(+0)$ exist, that $F^{\prime}(x)$ exist almost everywhere on $I$ and be in class $\Sigma_{x}$, and that for all $x$ in $I^{0}, F(x)=F(+0)+\int_{0}^{x} F^{\prime}(u) d u$. The sufficiency is obvious. If $F$ is in $A_{x}$, then $F_{1}^{\prime}(x)$ exists almost everywhere in every $I_{X}$ and ( $\left.{ }^{2}\right)$ $F_{1}(x)=F_{1}(0)+\int_{0}^{x} F_{1}^{\prime}(u) d u$. If $S$ is the set of points on $I^{0}$ where $F^{\prime}(x)=F_{1}^{\prime}(x)$ exists, then $m(I-S)=\lim _{n} m\left(I_{n}-S \cdot I_{n}\right)=\lim _{n} 0=0$. Hence, $F^{\prime} \in \Sigma_{x}$ and the necessity is proved.

When $F(x)$ is in class $A_{x}$ and $s$ is any complex number, the following formula for integration by $\operatorname{parts}\left({ }^{3}\right)$ holds for $X>0$,

$$
\int_{0}^{x} e^{-s x} F^{\prime}(x) d x=e^{-s X} F(X)-F(+0)+s \int_{0}^{x} e^{-s x} F(x) d x .
$$

In particular class $A_{x}$ includes the following important types of func$\operatorname{tions}\left({ }^{4}\right)$ :

(1) $F(x)$ is continuous on $I^{0} ; F^{\prime}(x)$ exists and is finite except for a denumerable number of points and is in class $\Sigma_{x}$,

(2) $F^{\prime}(x)$ exists for all $x>0$ and belongs to $\Sigma_{x}$,

(3) $F(x)$ is continuous on $I^{0}$ and $F^{\prime}(x)$ is sectionally continuous,

(4) $F^{\prime}(x)$ exists for all $x>0$ and is bounded in every $I_{X}^{0}: 0<x<X$,

(5) $F(x)$ satisfies a Lipschitz condition in every $I_{X}^{0}$, that is, there exists a constant $N_{X}$ such that for all $x$ and $x^{\prime}$ in $I_{X}^{0}$

$$
\left|F(x)-F\left(x^{\prime}\right)\right| \leqq N_{X}\left|x-x^{\prime}\right| \text {, }
$$

(6) The four derivates $D^{+} F, D_{+} F, D^{-} F, D_{-} F$ are bounded in every $I_{X}^{0}$. This is equivalent to (5).

The extension of class $\Sigma_{x}$ to functions of two variables is immediate. A function $F(x, y)$ defined on a measurable kernel $W$ of the quadrant $Q$ : $0 \leqq x<\infty, 0 \leqq y<\infty$ is said to be of class $\Sigma$ if the equivalent function $F_{0}$ defined in $Q$ by

$$
F_{0}(x, y)=F(x, y) \text { on } W ; \quad F_{0}(x, y)=0 \text { on } Q-W
$$

is Lebesgue summable in every finite rectangle $R_{X Y}: 0 \leqq x \leqq X, 0 \leqq y \leqq Y$. By $\int_{(0,0)}^{(X, Y)} F(x, y) d(x, y)$ we mean $\int_{R_{X Y}} F_{0}(x, y) d(x, y)$ which is equal to $\int_{W \cdot R_{X Y}} F(x, y) d(x, y)$. For brevity, if $F(x, y)$ is of class $\Sigma$ and is of class $\Sigma_{x}$

(2) E. J. McShane, Integration, Princeton, Princeton University Press, 1944 pp. 159, 208.

(8) Ibid., p. 209. This gives the formula for $F_{1}$.

(4) That the type of function defined in (1) is in class $A_{x}$ may be found in C. Caratheodory, Vorlesungen ïber reele Funktionen, 2d Ed. Leipzig, Teubner, 1927, p. 597. (2), (3), (4) are special cases of (1). That the type of function defined in (5) is in class $A_{x}$ follows from the proof on page 50 of McShane, and that (6) is equivalent to (5) from McShane, page 207. 
for every $y$ and of class $\Sigma_{y}$ for every $x$, we say that $F$ is of class $\overline{\boldsymbol{\Sigma}}$. The following lemma, which is an extension of Fubini's theorem, says that functions of class $\Sigma$ can be replaced essentially by functions of class $\bar{\Sigma}$. By $J$ we mean the interval $0 \leqq y<\infty$.

Lemma 1. Let $F$ be defined on $W$ and belong to class $\Sigma$. Then there exist measurable kernels $S$ and $T$ of $I$ and $J$ respectively such that each of the following functions is in class $\bar{\Sigma}$ :

$$
\begin{aligned}
& \bar{F}(x, y)=F(x, y) \text { on }(S \times T) W \\
& \bar{F}(x, y)=0 \text { on } Q-(S \times T) W
\end{aligned}
$$

$\Theta(x, y)=\int_{0}^{x} \bar{F}(u, y) d u$

$$
\begin{aligned}
& \Psi(x, y)=\int_{0}^{y} \bar{F}(x, v) d v \\
& \Phi(x, y)=\int_{(0,0)}^{(x, y)} F(u, v) d(u, v)=\int_{(0,0)}^{(x, y)} \bar{F}(u, v) d(u, v) .
\end{aligned}
$$

Everywhere in $Q$

$$
\Phi(x, y)=\int_{0}^{x} \Psi(u, y) d u=\int_{0}^{y} \Theta(x, v) d v .
$$

Proof. Since $F_{0}$ is summable in every finite rectangle $R_{X Y}$, by Fubini's theorem (5) $F_{0}$ is a summable function of $y$ on $J_{Y}$ for all $x$ in $S_{X Y} \subseteq I_{X}$ where $m\left(I_{X}-S_{X Y}\right)=0$. For a fixed $X$ let $Y$ assume positive integral values; if $F_{0}$ is summable on $J_{n+1}$, it is summable on $J_{n}$ and, hence, $S_{X, n+1} \subseteq S_{X, n}$. Thus $S_{X}=\lim _{n} S_{X, n}$ is the set of all $x$ in $I_{X}$ for which $F_{0}$ is summable in every $J_{n}$, that is, for which $F_{0} \in \Sigma_{y}$ and $m\left(I_{X}-S_{X}\right)=0$ (since $I_{X}-S_{X}=\lim _{n}\left(I_{X}-S_{X, n}\right)$ ).

Now let $X$ assume positive integral values. Then $\left\{S_{n}\right\}$ is an increasing sequence of sets and $S=\lim _{n} S_{n}=\Sigma S_{n}$ is the set of all $x$ in $I$ for which $F_{0} \in \Sigma_{y}$. Since $I-S=\lim _{n}\left(I_{n}-S_{n}\right), m(I-S)=0$.

In a similar manner one finds a measurable kernel $T$ of $J$ such that $F_{0} \in \Sigma_{x}$ whenever $y \in T$. If $\bar{F}$ is defined by (1.2), $F_{0}$ and $\bar{F}$ differ only on a twodimensional set of measure 0 so that $\bar{F} \in \Sigma$. Whenever $x \in S, \bar{F}$ and $F_{0} \operatorname{differ}$ only on a one-dimensional set of measure 0 so that $\bar{F} \in \Sigma_{y}$; when $x \in I-S$, $\bar{F} \equiv 0$ and hence $\bar{F} \in \Sigma_{y}$. Likewise $\bar{F}$ is in class $\Sigma_{x}$ for every $y$.

Hence, the functions $\theta, \psi$, and $\phi$ can be defined in $Q$ as in (1.3)-(1.5). It follows from Fubini's theorem that in every finite rectangle $\Theta(x, y)$ is a summable function of $y$ for every $x$ and that $\Phi(x, y)=\int_{0}^{y} \Theta(x, v) d v$. Hence, $\Theta \in \Sigma_{y}$ for every $x$ and similarly $\Psi \in \Sigma_{x}$ for every $y$. Thus (1.6) holds in every $R_{X Y}$

(5) McShane, p. 137. 
and consequently in $Q$. Since $\Theta$ is an absolutely continuous function of $x$ in every $I_{X}$, it certainly is in class $\Sigma_{x}$ for every $y$. By a result of Carathéodory $\left(^{6}\right) \Theta(x, y)$ is a measurable function of both variables in every $R_{X Y}$; the same is true of $\Theta(x, y)=\int_{0}^{x}|\bar{F}(u, y)| d u$. Now $\Phi(x, y)$ $=\int_{(0,0)}^{(x, y)}|F(u, v)| d(u, v)$ exists and equals $\int_{0}^{y} \bar{\Theta}(x, v) d v$. Therefore $\left({ }^{7}\right), \int_{0}^{x} \bar{\Phi}(u, y) d u$ exists and by Tonelli's theorem $\bar{\Theta}(x, y)$ is summable in $R_{X Y}$. By the comparison test $\Theta$ is summable in $R_{X Y}$ and thus is in class $\Sigma$. The function $\Phi(x, y)$, being absolutely continuous in both variables in every $R_{X Y}$, is certainly in $\bar{\Sigma}$.

In particular, if $F(x, y)$ is defined in $Q$ and belongs to $\bar{\Sigma}$, then $W=Q$, $S=I, T=J$, and $\bar{F}=F$ in $Q$.

If $F \in \Sigma$ and $\bar{F}$ is defined as in (1.2), it follows immediately from Lemma 1 that the functions

$$
\begin{aligned}
f_{1}(x, y ; s) & =\int_{0}^{x} e^{-s u} \bar{F}(u, y) d u, \\
f_{2}(x, y ; t) & =\int_{0}^{y} e^{-t v} \bar{F}(x, v) d v, \\
f(x, y ; s, t) & =\int_{(0,0)}^{(x, y)} e^{-s u-t v} F(u, v) d(u, v)
\end{aligned}
$$

are all in class $\overline{\mathbf{\Sigma}}$. Everywhere in $Q$

$$
f(x, y ; s, t)=\int_{0}^{y} e^{-t v} f_{1}(x, v ; s) d v=\int_{0}^{x} e^{-s u} f_{2}(u, y ; t) d u .
$$

The following lemma gives formulas for integration by parts:

Lemma 2. Let $F \in \Sigma$ and define $f_{1}, f_{2}, f$ as in (1.7). Then everywhere in $Q$

$$
\begin{aligned}
f_{1}(X, y ; s+h)= & e^{-h X} f_{1}(X, y ; s)+h \int_{0}^{X} e^{-h x} f_{1}(x, y ; s) d x, \\
f_{2}(x, Y ; t+k)= & e^{-k Y} f_{2}(x, Y ; t)+k \int_{0}^{Y} e^{-k y} f_{2}(x, y ; t) d y, \\
f(X, Y ; s+h, t)= & e^{-h X} \int_{0}^{Y} e^{-t y} f_{1}(X, y ; s) d y \\
& +h \int_{(0,0)}^{(X, Y)} e^{-h x-t y} f_{1}(x, y ; s) d(x, y) \\
= & e^{-h X} f(X, Y ; s, t)+h \int_{0}^{X} e^{-h x} f(x, Y ; s, t) d x,
\end{aligned}
$$

(6) Caratheodory, p. 656.

(?) McShane, pp. 126, 145. 


$$
\begin{aligned}
f(X, Y ; s, t+k)= & e^{-k Y} \int_{0}^{X} e^{-s x} f_{2}(x, Y ; t) d x \\
& +k \int_{(0,0)}^{(X, Y)} e^{-s x-k y} f_{2}(x, y ; t) d(x, y) \\
= & e^{-k Y} f(X, Y ; s, t)+k \int_{0}^{Y} e^{-k y} f(X, y ; s, t) d y, \\
f(X, Y ; s+h, t+k)= & e^{-h X-k Y} f(X, Y ; s, t) \\
& +h e^{-k Y} \int_{0}^{X} e^{-h x} f(x, Y ; s, t) d x \\
& +k e^{-h X} \int_{0}^{Y} e^{-k y} f(X, y ; s, t) d y \\
& +h k \int_{(0,0)}^{(X, Y)} e^{-h x-k y} f(x, y ; s, t) d(x, y) .
\end{aligned}
$$

Proof. Since $f_{1}(x, y ; s)$ is an integral, it is absolutely continuous in $x$ in every finite interval $I_{x}$ and almost everywhere $\partial f_{1} / \partial x=e^{-s x} \bar{F}(x, y)$. Applying (1.1) with $h$ instead of $s$, we have

$$
\begin{aligned}
f_{1}(X, y ; s+h) & =\int_{0}^{x} e^{-h x} \cdot e^{-s x} \bar{F}(x, y) d x \\
& =e^{-h x} f_{1}(X, y ; s)+h \int_{0}^{x} e^{-h x} f_{1}(x, y ; s) d x
\end{aligned}
$$

which is (1.9). Multiplying both sides of (1.9) by $e^{-t y}$, integrating from 0 to $Y$, and replacing the repeated integral by a double integral, we obtain from Lemma 1

$$
\begin{aligned}
\int_{0}^{Y} e^{-t y} f_{1}(X, y ; s+h) d y= & e^{-h X} \int_{0}^{Y} e^{-t y} f_{1}(X, y ; s) d y \\
& +h \int_{(0,0)}^{(X, Y)} e^{-h x-t y} f_{1}(x, y ; s) d(x, y) .
\end{aligned}
$$

The left-hand side of this equation is just $f(X, Y ; s+h, t)$ by $(1.8)$; so the first part of (1.11) follows. On substituting again from (1.8) on the right side, the first term is just $e^{-h X} f(X, Y ; s, t)$ and the second is

$$
h \int_{0}^{x} e^{-h x} d x \int_{0}^{Y} e^{-t y} f_{1}(x, y ; s) d y=h \int_{0}^{x} e^{-h x} f(x, Y ; s, t) d x,
$$

which yields the second part of (1.11). Formulas (1.10) and (1.12) are estab- 
lished in a similar fashion. To get (1.13), first use (1.12) with $s+h$ instead of $h$ : $f(X, Y ; s+h, t+k)=e^{-k Y} f(X, Y ; s+h, t)+k \int_{0}^{Y} e^{-k y} f(X, y ; s+h, t) d y$

and then substitute from (1.11) in the right-hand side. The final term in the result is a repeated integral which can be replaced by a double integral since $f \in \bar{\Sigma}$.

Let $F(x, y)$ be defined in $Q^{0}: 0<x<\infty, 0<y<\infty$. A necessary and sufficient condition that $F$ be in class $A_{x}$ for every $y>0$ is that for every $y>0$, $G(y)=F(+0, y)$ exist, that for every $y>0, F_{x}$ exist for almost all $x \in I$ and $F_{x} \in \Sigma_{x}$ and that everywhere in $Q^{0}$

$$
F(x, y)=G(y)+\int_{0}^{x} F_{x}(u, y) d u .
$$

One extension of class $A_{x}$ to two variables (an analogue of absolute continuity for finite rectangles as defined by Tonelli) is the following: $F$ is in class $A^{*}$ if $F$ is defined in $Q^{0}, F \in A_{x}$ for every $y>0, F \in A_{y}$ for every $x>0$, and $F_{x}$ and $F_{y}$ belong to $\Sigma$.

Lemma 3.(a) Let $F(x, y) \in A_{x}$ for every $y>0$, let $F_{x} \in \Sigma$, and $G(y) \in \Sigma_{y}$. Then $F(x, y)$ is in class $\bar{\Sigma}$ and on defining

$$
M(x, y)=\int_{0}^{\nu} \overline{F_{x}}(x, v) d v, \quad \Psi(x, y)=\int_{0}^{y} F(x, v) d v, \quad G_{0}(y)=\int_{0}^{y} G(v) d v,
$$

$M$ and $\Psi$ are in $\bar{\Sigma}$,

$$
\Psi(x, y)=G_{0}(y)+\int_{(0,0)}^{(x, y)} F_{x}(u, v) d(u, v)=G_{0}(y)+\int_{0}^{x} M(u, y) d u,
$$

and $\Psi(+0, y)=G_{0}(y)$.

(b) Let $F(x, y) \in A_{y}$ for every $x>0$, let $F_{y} \in \Sigma$, and $H(x)=F(x,+0) \in \Sigma_{x}$. Then $F(x, y) \in \bar{\Sigma}$ and

$$
F(x, y)=B(x)+\int_{0}^{y} F_{y}(x, v) d v .
$$

\section{On defining}

$$
N(x, y)=\int_{0}^{x} \overline{F_{y}}(u, y) d u, \Theta(x, y)=\int_{0}^{x} F(u, y) d u, \quad H_{0}(x)=\int_{0}^{x} B(u) d u,
$$

$N$ and $\Theta$ are in $\bar{\Sigma}$,

$$
\Theta(x, y)=H_{0}(x)+\int_{(0,0)}^{(x, y)} F_{y}(u, v) d(u, v)=H_{0}(x)+\int_{0}^{v} N(x, v) d v
$$


and $\Theta(x,+0)=H_{0}(x)$.

(c) If $F(x, y)$ belongs to $A^{*}$, then $G(y) \in \Sigma_{y}, H(x) \in \Sigma_{x}$, the functions $F, M, N, \Theta$, and $\Psi$ are in $\bar{\Sigma}, \Psi(+0, y)=G_{0}(y), \Theta(x,+0)=H_{0}(x)$, and formulas (1.14)-(1.17) hold.

Proof. (a) Apply Lemma 1 to $F_{x}$ instead of $F$. Then $M(x, y), \int_{0}^{x} \bar{F}_{x}(u, y) d u$, $\int_{(0,0)}^{(x, y)} F_{x}(u, v) d(u, v)$ all belong to class $\bar{\Sigma}$ and

$$
\int_{(0,0)}^{(x, y)} F_{x}(u, v) d(u, v)=\int_{0}^{x} M(u, y) d u=\int_{0}^{y}\left[\int_{0}^{x} \overline{F_{x}}(u, v) d u\right] d v .
$$

For all $y>0, F_{x}$ is defined for almost all $x \in I$ and, hence, the set $T$ of the lemma becomes $J$ itself. Hence, $\int_{0}^{x} \bar{F}_{x}(u, y) d u=\int_{0}^{x} F_{x}(u, y) d u=F(x, y)-G(y)$ by (1.14), and so $F \in \bar{\Sigma}$. Formula (1.15) easily follows.

(c) Formula (1.14) holds and $\int_{0}^{x} F_{x}(u, y) d u$ belongs to $\bar{\Sigma}$. Now $G(y)$ $=F(x, y)-\int_{0}^{x} F_{x}(u, y) d u$ is the difference of two functions of class $\Sigma_{y}$ and so belongs to $\Sigma_{y}$. Likewise $H(x) \in \Sigma_{x}$. Then apply the first two parts of the lemma.

Another extension of class $A_{x}$ to two variables (the analogue of absolute continuity for finite rectangles as defined by Carathéodory) is the following: A function $F(x, y)$ defined in $Q^{0}$ is of class A if $F(+0, y)=G(y), F(x,+0)$ $=H(x), F(+0,+0)=c_{0}$ all exist and the function $F_{1}(x, y)$, defined by the equations

$F_{1}(x, y)=F(x, y)$ in $Q^{0} ; F_{1}(0, y)=G(y)$ for $y>0, F_{1}(x, 0)=H(x)$ for $x>0$,

$$
F_{1}(0,0)=c_{0} \text {, }
$$

is absolutely continuous, as defined by Carathéodory $\left(^{8}\right)$, in every $R_{X Y}$. From the necessary and sufficient condition given by Carathéodory it follows that a necessary and sufficient condition that $F(x, y)$ be in class $\mathrm{A}$ is that there exist functions $K(x, y), P(x), Q(y)$ all in $\Sigma$ and a constant $c_{0}$ such that

$$
F(x, y)=\int_{(0,0)}^{(x, y)} K(u, v) d(u, v)+\int_{0}^{x} P(u) d u+\int_{0}^{y} Q(v) d v+c_{0}
$$

It is no loss of generality to assume that $K$ is defined in $Q$ and belongs to $\bar{\Sigma}$. (See Lemma 1.) The function $\phi$ of Lemma 1 and the functions $\psi$ and $\theta$ given by formulas (1.15) and (1.17) of Lemma 3 are in class A.

LEMma 4. Let $F(x, y) \in A$. Then (1.19) holds and

$$
\begin{aligned}
& G(y)=\int_{0}^{y} Q(v) d v+c_{0}=\int_{0}^{y} G^{\prime}(v) d v+c_{0}, \\
& H(x)=\int_{0}^{x} P(u) d u+c_{0}=\int_{0}^{x} H^{\prime}(u) d u+c_{0} .
\end{aligned}
$$

(8) Caratheodory, pp. 653-654. 
Furthermore $F(x, y) \in A^{*}$ so that all statements and formulas of Lemma 3(c) hold. There are measurable kernels $S_{3}$ of $I$ and $T_{3}$ of $J$ such that $F_{x}$ exists on $S_{3} \times J$ and $F_{y}$ exists on $I \times T_{3}$ and $F_{x y}=F_{y x}=K$ on at least the measurable kernel $S_{3} \times T_{3}$.

Set $\overline{F_{x y}}=K$ on $S_{3} \times T_{3}$ and 0 elsewhere. For $a$ fixed $y \in T_{3}, \overline{F_{x y}}=F_{y x}$ for almost all $x$ and on $I \times T_{3}$

(1.20) $F_{y}(x, y)-G^{\prime}(y)=\int_{0}^{x} K(u, y) d u=\int_{0}^{x} \overline{F_{x y}}(u, y) d u=\int_{0}^{x} F_{y x}(u, y) d u$.

For a fixed $x \in S_{3}, F_{x y}=\overline{F_{x y}}$ for almost all $y$ and on $S_{3} \times J$

$$
F_{x}(x, y)-H^{\prime}(x)=\int_{0}^{y} K(x, v) d v=\int_{0}^{y} \overline{F_{x y}}(x, v) d v=\int_{0}^{y} F_{x y}(x, v) d v
$$

Everywhere in $Q$

$$
\begin{array}{rlrl}
F(x, y) & =\int_{(0,0)}^{(x, y)} \overline{F_{x y}}(u, v) d(u, v)+\int_{0}^{x} H^{\prime}(u) d u+\int_{0}^{y} G^{\prime}(v) d v+c_{0}, \\
G^{\prime}(y) & =\lim _{x \rightarrow 0+} F_{y}(x, y) & \text { on } T_{3}, \\
H^{\prime}(x) & =\lim _{y \rightarrow 0+} F_{x}(x, y) & \text { on } S_{3} .
\end{array}
$$

Proof. When $F \in A$, (1.19) holds, whence $G(y)=\int_{0}^{\nu} Q(v) d v+c_{0}$ is of class $\mathrm{A}_{y}$ so that by the one-dimensional result $G^{\prime}(y)$ exists and equals $Q(y)$ on a measurable kernel $T_{1}$ of $J$. If $L(x, y)=\int_{(0,0)}^{(x, y)} K(u, v) d(u, v)$, then we shall prove that

$$
L_{y}(x, y) \text { exists and equals } \int_{0}^{x} K(u, y) d u
$$

for all $y \in T_{1}$ and all $x$. To do this, one uses the corresponding result proved by Fubini for a finite rectangle $\left({ }^{9}\right)$ : in our notation that for every $R_{X Y}$ there exists a measurable kernel $T_{X Y} \subseteq J_{Y}$ such that (1.25) holds on $I_{X} \times T_{X Y}$. As in Lemma 1 we show that $T_{2}=\lim _{m} \lim _{n} T_{m n}$ is a measurable kernel of $J$. Hence, for all $x$ and for $y \in T_{3}=T_{1} T_{2}, F_{y}$ exists and

$$
F_{y}(x, y)=\int_{0}^{x} K(u, y) d u+G^{\prime}(y) \quad \text { on } I \times T_{3} .
$$

By Lemma 1 the integral on the right is in $\bar{\Sigma}$ and $G^{\prime}(y) \in \Sigma_{y}$. Hence, $F_{y} \in \Sigma$ and $\Sigma_{y}$, and $F_{y} \in \Sigma_{x}$ for $y \in T_{3}$. Also, from (1.19),

(9) This result was first published in correspondence between Fubini and Tonelli appearing in Rend. Circ. Mat. Palermo vol. 40 (1915) pp. 297-298. It was called to the authors' attention by Professor Marston Morse. 


$$
\begin{aligned}
\int_{0}^{y} F_{y}(x, v) d v & =\int_{0}^{y} d v \int_{0}^{x} K(u, v) d u+\int_{0}^{y} G^{\prime}(v) d v \\
& =\int_{(0,0)}^{(x, y)} K(u, v) d(u, v)+G(y)-c_{0}=F(x, y)-H(x) .
\end{aligned}
$$

Hence, $F \in A_{y}$ for every $x>0$. Statements about $F_{x}$ are established in parallel fashion. But then $F \in A^{*}$ so that all results of Lemma 3(c) hold.

From (1.26) for $y \in T_{3}, F_{x y}$ exists and equals $K(x, y)$ for $x \in S^{\nu}$, a measurable kernel of $I$. Likewise for $x \in S_{3}, F_{x y}$ exists and equals $K(x, y)$ for $y \in T^{x}$, a measurable kernel of $J$. Now set $\overline{F_{x y}}=K$ on $S_{3} \times T_{3}$ and 0 elsewhere. Thus for a fixed $y \in T_{3}, \overline{F_{x y}}=K=F_{y x}$ for almost all $x$, that is, for $x \in S_{3} \cdot S^{y}$ and thus from (1.26) formula (1.20) holds. The second statement is proved in parallel fashion, and the rest of the theorem follows immediately.

On applying Lemma 3(a) to $F_{x}$ instead of $F$, we easily get the following:

Lemma 5. Let $F_{x}$ belong to $A_{x}$ for every $y>0$; let $F_{x x} \in \Sigma$; let $G_{1}(y)$ $=\lim _{x \rightarrow 0+} F_{x}(x, y)=F_{x}(+0, y)$ and $G(y) \in \Sigma_{y}$. Then $F, F_{x}, M, V(x, y)$ $=\int_{0}^{\nu} \overline{F_{x x}}(x, v) d v$ all belong to $\bar{\Sigma} ;$ indeed, $M \in A, F \in A_{x}$, and $V \in A_{y}$.

If we apply the last part of Lemma 3 to $F_{x}$ and $F_{y}$, we obtain:

Lemma 6. Let $F_{x}$ and $F_{y}$ be in $A^{*}$. Then $F(x, y), M(x, y), N(x, y), G(y)$, $H(x)$ are all in class $\mathrm{A} ; V \in A_{y}, U(x, y)=\int_{0}^{x} \overline{F_{y y}}(u, y) d u$ belongs to $A_{x}, G_{1}(y)$ $=F_{x}(+0, y), H_{2}(x)=F_{y}(x,+0), H_{1}(x)=F_{x}(x,+0), G_{2}(y)=F_{y}(+0, y)$ are all in $\bar{\Sigma}$. Formulas (1.14)-(1.24) hold.

Proof. Applying Lemma 3(c) first to $F_{x}$ and then to $F_{y}$, we find that $F_{x}, F_{y}, H_{1}, H_{2}, G_{1}, G_{2}$ all belong to $\bar{\Sigma}$, that $M, N, \int_{0}^{x} F_{x}(u, y) d u, \int_{0}^{y} F_{y}(x, v) d v$ belong to $\mathrm{A}$, and that $\int_{0}^{x} H_{1}(u) d u=\lim _{y \rightarrow 0+} \int_{0}^{x} F_{x}(u, y) d u, \int_{0}^{y} G_{2}(v) d v$ $=\lim _{x \rightarrow 0+} \int_{0}^{y} F_{y}(x, v) d v$. Since for every $y>0, F_{x}$ exists and belongs to $\Sigma_{x}$, $F \in A_{x}$. Similarly, $F \in A_{y}$. Hence, $F \in A^{*}$ and by Lemma $3(\mathrm{c})$ formulas (1.14)-(1.17) hold. We can now apply Lemma 5 and the parallel lemma to find that $V \in A_{y}$ and $U \in A_{x}$.

Since $\int_{0}^{x} F_{x}(u, y) d u$ is in class $\mathrm{A}$, it is in class $\mathrm{A}_{y}$ for every $x>0$. By (1.14) $G(y)$ being the difference of two functions of class $\mathrm{A}_{y}$ is also in class $\mathrm{A}_{y}$. Considered as a function of two variables, this means that $G(y) \in \mathrm{A}$. By (1.14), $F(x, y)$ is the sum of two functions of class A, and so is also of class A. Hence, we can apply Lemma 4.

The following two formulas are consequences of Lemma 3(c) applied to $F_{x}$ and $F_{y}$ and are occasionally useful.

$$
\begin{aligned}
& F_{x}(x, y)=G_{1}(y)+\int_{0}^{x} F_{x x}(u, y) d u=H_{1}(x)+\int_{0}^{y} F_{x y}(x, v) d v \\
& F_{y}(x, y)=H_{2}(x)+\int_{0}^{y} F_{y y}(x, v) d v=G_{2}(y)+\int_{0}^{x} F_{y x}(u, y) d u .
\end{aligned}
$$


2. Types of convergence and transformation of integrals. Let $F(x, y) \in \Sigma$ and set

$$
f(X, Y ; s, t)=\int_{(0,0)}^{(X, Y)} e^{-s x-t y} F(x, y) d(x, y)
$$

where $s$ and $t$ are two independent complex parameters

$$
s=\sigma+i \lambda, \quad t=\tau+i \mu .
$$

If $\lim _{X, Y} f(X, Y ; s, t)$ exists as $X, Y \rightarrow \infty$ simultaneously but independently, we say that the double Laplace transform

$$
\int_{Q} e^{-s x-t y} F(x, y) d(x, y)
$$

converges at $(s, t)$ and has the value $f(s, t)=\lim _{X, Y} f(X, Y ; s, t)$. If, in addition, there exists an $M$ independent of $X$ and $Y$ such that

$$
|f(X, Y ; s, t)| \leqq M \quad \text { for all }(X, Y) \in Q,
$$

then the integral is said to converge boundedly at $(s, t)$.

That a convergent integral need not converge boundedly is illustrated by the following example. The double Laplace transform of the function $F$ defined by

$$
\begin{aligned}
& F(x, y)=\left\{\begin{aligned}
0 & \text { for } \quad(0 \leqq x \leqq 2,0 \leqq y \leqq 2) \text { and }(x>2, y>2), \\
x & \text { for }(x>2,0 \leqq y \leqq 1), \\
-x & \text { for } \quad(x>2,1<y \leqq 2),
\end{aligned}\right. \\
& F(x, y)=F(y, x)
\end{aligned}
$$

converges, but not boundedly, at the point $s=0, t=0$.

If the integral (2.1) converges uniformly in $D$ and if there exists a constant $M_{0}$ such that $|f(X, Y ; s, t)| \leqq M_{0}$ for all $(X, Y) \in Q$ and all $(s, t) \in D$, then the integral is said to converge uniformly boundedly in $D$.

By $L\{F\}$ we mean the operation of forming the integral (2.1). As indicated above, we shall use small letters for transforms and capital letters for object functions. The symbol $D(a ; b)$ denotes the set of points $(s, t)$ with $R(s)>a, R(t)>b$, and $D[a ; b]$ its closure.

In the four theorems following we collect results which were proved independently by D. L. Bernstein( $\left.{ }^{1}\right)$ and L. Amerio( $\left.{ }^{1}\right)$.

THEOREM 2.1. If $L\{F\}$ converges boundedly at $\left(s_{0}, t_{0}\right)$, then

(a) $L\{F\}$ converges boundedly for all $(s, t)$ in $D\left(\sigma_{0} ; \tau_{0}\right)$.

(b) If $H$ and $K$ are any numbers greater than one and if $M$ is an upper bound of $\left|f\left(X, Y ; s_{0}, t_{0}\right)\right|$, then 


$$
|f(s, t)|<M H K \quad \text { and } \quad|f(X, Y ; s, t)|<4 M H K
$$

for all $(s, t)$ in $D_{1}:\left|s-s_{0}\right|<H\left(\sigma-\sigma_{0}\right),\left|t-t_{0}\right|<K\left(\tau-\tau_{0}\right)$.

(c) For any positive numbers $\delta_{1}, \delta_{2}$ and for any $H, K$ greater than one, $L\{F\}$ converges uniformly boundedly in

$$
\begin{aligned}
& D_{2}:\left|s-s_{0}\right|<H\left(\sigma-\sigma_{0}\right) \quad \sigma \geqq \sigma_{0}+\delta_{1}, \\
& \left|t-t_{0}\right|<K\left(\tau-\tau_{0}\right) \quad \tau \geqq \tau_{0}+\delta_{2} .
\end{aligned}
$$

(d) For all $(s, t) \in D\left(\sigma_{0} ; \tau_{0}\right), f(s, t)$ is an analytic function.

(e) For all $(s, t) \in D\left(\sigma_{0} ; \tau_{0}\right)$,

$$
\frac{\partial^{h+k} f(s, t)}{\partial s^{h} \partial t^{k}}=p(s, t)
$$

where $L\{P\}$ converges boundedly in $D\left(\sigma_{0} ; \tau_{0}\right)$ and

$$
P(x, y)=(-x)^{h}(-y)^{k} F(x, y) .
$$

Theorem 2.2. Let $\Phi(x, y)$ be defined in $Q$ by

$$
\Phi(x, y)=\int_{(0,0)}^{(x, y)} F(u, v) d(u, v) .
$$

If $L\{F\}$ converges boundedly at $\left(s_{0}, t_{0}\right)$, then there exists a constant $M_{1}$ such that

$$
|\Phi(x, y)| \leqq M_{1} e^{a x+b y} \quad \text { in } Q
$$

where $a=\max \left(\sigma_{0}, 0\right)$ if $s_{0} \neq i \lambda_{0}, a=\left|\lambda_{0}\right|$ if $s_{0}=i \lambda_{0}$, and $b=\max \left(\tau_{0}, 0\right)$ if $t_{0} \neq i \mu_{0}, b=\left|\mu_{0}\right|$ if $t_{0}=i \mu_{0}$.

If $\Phi(x, y)$ is of exponential type, that is, if there exist constants $M, a$, and $b$ such that

$$
|\Phi(x, y)| \leqq M e^{a x+b y}
$$

in $Q$,

$L\{F\}$ converges boundedly in $D(a ; b)$.

It is not true in general that the region of bounded convergence of $L\{F\}$ has the form $D(a ; b)$ as it does, say, when $F$ is a constant. One can characterize this region either by "associated abscissae of convergence" or by considering the properties of the four-dimensional regions involved. We shall not enter into a discussion of these concepts inasmuch as they are of no immediate use to us.

Further consequences of the hypothesis of Theorem 2.1 will be given in the corollary of Theorem 2.9.

THEOREM 2.3. Let $L\{F\}$ converge absolutely at $\left(s_{0}, t_{0}\right)$, that is, suppose that $\int_{Q}\left|e^{-s_{0} x-t_{0} y} F(x, y)\right| d(x, y)$ converges to a finite value $A$. Then

(a) $L\{F\}$ converges absolutely and uniformly boundedly in $D\left[\sigma_{0} ; \tau_{0}\right]$.

(b) $|f(X, Y ; s, t)| \leqq A$ for all $(X, Y) \in Q$ and $|f(s, t)| \leqq A$ in $D\left[\sigma_{0} ; \tau_{0}\right]$.

(c) $f(s, t)$ is an analytic function in $D\left(\sigma_{0} ; \tau_{0}\right)$. 
TheOREM 2.4. Let $\bar{\Phi}(x, y)$ be defined by

$$
\bar{\Phi}(x, y)=\int_{(0,0)}^{(x, y)}|F(u, v)| d(u, v) .
$$

If $L\{F\}$ converges absolutely at $\left(s_{0}, t_{0}\right)$, then $\bar{\Phi}(x, y) \leqq A e^{a x+b y}$ in $Q$ where $a=\max \left(\sigma_{0}, 0\right), b=\max \left(\tau_{0}, 0\right)$.

If $\bar{\Phi}(x, y)$ is of exponential type: $\bar{\Phi}(x, y) \leqq M e^{a x+b y}$ in $Q$, then $L\{F\}$ converges absolutely in $D(a ; b)$.

In particular, if $F(x, y)$ is summable in $Q$, then $L\{F\}$ converges absolutely in $D[0 ; 0]$.

Let $F \in \Sigma$ and define $f_{1}(X, y ; s)$ as in (1.7). If $\lim _{X \rightarrow \infty} f_{1}(X, y ; s)$ exists for a given $s$ and $y$, we call this the one-dimensional transform of $F$ and denote it by $L_{s}\{F\}$, that is,

$$
L_{s}\{F\}=\int_{0}^{\infty} e^{-s x} \bar{F}(x, y) d x
$$

whenever this integral converges; its value is $f_{1}(y ; s)=\lim _{X \rightarrow \infty} f_{1}(X, y ; s)$. Similarly, we define

$$
L_{t}\{F\}=\int_{0}^{\infty} e^{-t y} \bar{F}(x, y) d y
$$

whenever this integral converges; its value is $f_{2}(x ; t)=\lim _{Y \rightarrow \infty} f_{2}(x, Y ; t)$. Now if $f_{1}(y ; s) \in \Sigma_{y}$ and if

$$
L_{t}\left\{f_{1}\right\}=\int_{0}^{\infty} e^{-t y} f_{1}(y ; s) d y
$$

converges, we call this the iterated transform $L_{t} L_{s}\{F\}$. Similarly, if $f_{2}(x ; t)$ $\in \Sigma_{x}$ and if $L_{s}\left\{f_{2}\right\}=\int_{0}^{\infty} e^{-s x} f_{2}(x ; t) d x$ converges, we call this the iterated transform $L_{s} L_{t}\{F\}$. The usual way of writing these is

$$
\begin{aligned}
& L_{t} L_{s}\{F\}=\int_{0}^{\infty} e^{-t y} d y \int_{0}^{\infty} e^{-s x} \bar{F}(x, y) d x, \\
& L_{s} L_{t}\{F\}=\int_{0}^{\infty} e^{-s x} d x \int_{0}^{\infty} e^{-t y} \bar{F}(x, y) d y .
\end{aligned}
$$

It may happen that the double transform $L\{F\}$ is equal to one or both of these iterated transforms but in general the convergence or bounded convergence of $L\{F\}$ does not imply the existence of $L_{s}\{F\}$ or $L_{t}\{F\}$ or either of the two iterated transforms above. For absolute convergence, on the other hand, we have the following:

ThEOREM 2.5. Let $L\{F\}$ converge absolutely at $(s, t)$. Then $L_{\mathbf{s}}\{F\}$, 
$L_{t}\{F\}, L_{s}\left\{f_{2}\right\}$, and $L_{t}\left\{f_{1}\right\}$ converge absolutely, and

$$
L\{F\}=L_{s} L_{t}\{F\}=L_{t} L_{s}\{F\} .
$$

Proof. Since $H(x, y)=e^{-\boldsymbol{s} x-t y} F_{0}(x, y)$ is summable on $Q$, by Fubini's theorem for the infinite rectangle there are measurable kernels $S \subseteq I$ and $T \subseteq J$ such that the function $\bar{H}=H$ on $S \times T$ and 0 elsewhere is summable in each variable for every value of the other and $A_{2}(y)=\int_{0}^{\infty} \bar{H}(x, y) d x$ is summable on $J$ with

$$
A=\int_{0}^{\infty} A_{2}(y) d y=\int_{Q} H(x, y) d(x, y) .
$$

Now defining $\bar{F}$ by (1.2) we see that $e^{-s x} \bar{F}(x, y)=e^{t y} \bar{H}(x, y)$ is summable on $I$ for every $y$, which means that $L_{s}\{F\}$ converges absolutely. Also, $f_{1}(y ; s)$ $=e^{t y} A_{2}(y)$. But then $e^{-t y} f_{1}(y ; s)=A_{2}(y)$ is summable on $J$ which means that $L_{t}\left\{f_{1}\right\}$ converges absolutely and $L_{t}\left\{f_{1}\right\}=A$. Since $L_{t}\left\{f_{1}\right\}=L_{t} L_{s}\{F\}$ and $A=L\{F\}$ by the definition of $H$, we have the desired equality. The other half is proved in parallel fashion.

Theorem 2.6. Let $F \in \Sigma$ and let

$$
|F(X, Y)| \leqq M e^{a X+b Y} \quad \text { for all }(X, Y) \in W .
$$

Then, in $D(a ; b), L_{s}\{F\}, L_{t}\{F\}, L\{F\}$ converge absolutely and (2.5) holds. Furthermore in $D(a ; b)$

$$
\begin{aligned}
\left|f_{1}(X, Y ; s)\right| & \leqq M_{0} e^{\tau Y}, & & \left|f_{1}(Y ; s)\right| \leqq M_{0} e^{\tau Y}, \\
\left|f_{2}(X, Y ; t)\right| & \leqq M_{0} e^{\sigma X}, & & \left|f_{2}(X ; t)\right| \leqq M_{0} e^{\sigma X}, \\
|f(X, Y ; s, t)| & \leqq M_{0} . & &
\end{aligned}
$$

Proof. We have $\left|e^{-s x-t y} \bar{F}(x, y)\right| \leqq M e^{-h_{1} x-k_{1} y}$ where $h_{1}=\sigma-a, k_{1}=\tau-b$. When $h_{1}>0, k_{1}>0, L\{F\}$ converges absolutely by comparison with $M \int_{Q} e^{-h_{1} x-k_{1} y} d(x, y)=M / h_{1} k_{1}$. The previous theorem then yields the absolute convergence of the single transforms and (2.5). Now for $h_{1}>0,\left|f_{1}(X, Y ; s)\right|$ $=\left|\int_{0}^{X} e^{-s x} \bar{F}(x, Y) d x\right| \leqq M e^{b Y} \int_{0}^{X} e^{-h_{1} x} d x \leqq\left(M / h_{1}\right) e^{b Y}=M_{2} e^{b Y}$. Hence, $\left|f_{1}(Y ; s)\right|$ $\leqq M_{1} e^{b Y}$. The other inequalities follow in similar fashion.

An important type of convergence which is more special than bounded convergence but more general than absolute convergence is the following: $L\{F\}$ is said to converge s-boundedly at $(s, t)$ if $L\{F\}$ converges boundedly, $L_{s}\{F\}$ converges for all $y$, and $\left|f_{1}(X, Y ; s)\right| \leqq M e^{\tau Y}$ in $Q$. Analogously, $L\{F\}$ is said to converge $t$-boundedly at $(s, t)$ if $L\{F\}$ converges boundedly, $L_{t}\{F\}$ converges for all $x$, and $\left|f_{2}(X, Y ; t)\right| \leqq M e^{\sigma X}$ in $Q$.

THEOREM 2.7. If $L\{F\}$ converges $s$-boundedly at $\left(s_{0}, t_{0}\right)$, then, in $D\left(\sigma_{0} ; \tau_{0}\right)$, $L\{F\}$ converges s-boundedly, $L_{t}\left\{f_{1}\right\}$ converges absolutely, $\left|f_{1}(y ; s)\right| \leqq M e^{r_{0} \nu}$ $\leqq M e^{r y}$, and $L\{F\}=L_{t} L_{s}\{F\}$. 
Proof. By Lemma 2 with $h=s-s_{0}$ and $G(X, Y)=f_{1}\left(X, Y ; s_{0}\right)$,

$$
f_{1}(X, y ; s)=e^{-h x} G(X, y)+h \int_{0}^{X} e^{-h x} G(x, y) d x .
$$

Since $\left|e^{-h x} G(x, y)\right| \leqq M e^{-\left(\sigma-\sigma_{0}\right) x+\tau_{0} y}$, the first term on the right approaches 0 and the second term approaches an absolutely convergent integral as $X \rightarrow \infty$ if $\sigma>\sigma_{0}$. Hence, $L_{s}\{F\}=\lim _{X \rightarrow \infty} f_{1}(X, y ; s)$ exists for $\sigma>\sigma_{0}$ and all $y$ and $f_{1}(y ; s)=h \int_{0}^{\infty} e^{-h x} G(x, y) d x$. Also, from the above, $\left|f_{1}(X, y ; s)\right| \leqq M_{1} e^{\tau_{0} y}$ and, hence, $\left|f_{1}(y ; s)\right| \leqq M_{1} e^{\tau_{0} y}$. For $\tau>\tau_{0}$, therefore, $L_{t}\left\{f_{1}\right\}$ converges absolutely.

We know that $f(X, Y ; s, t)=\int_{0}^{Y} e^{-t y} f_{1}(X, y ; s) d y$. Substituting for $f_{1}$ from equation (2.8) and using the fact that $\left|e^{-h x-t y} G(x, y)\right| \leqq M e^{-\left(\sigma-\sigma_{0}\right) x-\left(\tau-\tau_{0}\right) y}$, we find that $f(s, t)=h \int_{Q} e^{-h x-t y} G(x, y) d(x, y)$ which is an absolutely convergent integral. Thus

$$
\begin{aligned}
f(s, t) & =h \int_{0}^{\infty} e^{-t y} d y \int_{0}^{\infty} e^{-h x} G(x, y) d x=\int_{0}^{\infty} e^{-t y} f_{1}(y ; s) d y \\
& =L_{t}\left\{f_{1}\right\}=L_{t} L_{s}\{F\} .
\end{aligned}
$$

A comparison of this theorem with Theorem 2.3 and Theorem 2.5 shows that $s$-bounded convergence enjoys some of the properties of absolute convergence. A parallel theorem holds for $t$-bounded convergence.

It is convenient to say that $L\{F\}$ converges regularly at $(s, t)$ if $L\{F\}$ converges absolutely and $|F(x, y)| \leqq M e^{\sigma x+\tau y}$. If (2.6) holds, then $L\{F\}$ converges regularly (as well as $s$-boundedly and $t$-boundedly) in $D(a ; b)$.

The connection between the various types of convergence and functions of exponential type is not accidental but basic. Thus we have seen that the bounded convergence of $L\{F\}$ is essentially equivalent to the exponential typeness of its integral $\Phi(x, y)$. The following two theorems bring out the relationship of the transform of $F$ with the integrals $\Theta, \Psi$, and $\Phi$ defined in (1.3)-(1.5).

\section{THEOREM 2.8. Let $F \in \Sigma$ and let}

$$
\left|f_{1}\left(X, Y ; s_{1}\right)\right| \leqq M e^{a X+b Y}
$$

for all $(X, Y)$.

Set $\sigma_{0}=\sigma_{1}+a$. Then $L\{F\}$ converges s-boundedly in $D\left(\sigma_{0} ; b\right)$ and $L\{\Theta\}$ converges regularly in $D(c ; b)$ where $c=\max \left(\sigma_{0}, 0\right)$. Also in $D(c ; b)$

$$
f_{1}(y ; s)=s \theta_{1}(y ; s), \quad f(s, t)=s \theta(s, t) .
$$

Similarly, let

$$
\left|f_{2}\left(X, Y ; t_{1}\right)\right| \leqq M e^{a X+b Y}
$$

for $\operatorname{all}(X, Y)$.

Set $\tau_{0}=\tau_{1}+b$. Then $L\{F\}$ converges t-boundedly in $D\left(a ; \tau_{0}\right)$ and $L\{\Psi\}$ converges regularly in $D(a ; d)$ where $d=\max \left(0, \tau_{0}\right)$. Also in $D(a ; d)$

$$
f_{2}(x ; t)=t \psi_{2}(x ; t), \quad f(s, t)=t \psi(s, t) .
$$


Proof. Let $G(X, Y)=f_{1}\left(X, Y ; s_{1}\right)$ and $h=s-s_{1}$. Then (2.8) holds and $\left|e^{-h x} G(x, y)\right| \leqq M e^{-\left(\sigma-\sigma_{0}\right) x+b y}$. Proceeding as in the proof of Theorem 2.7, we find that $L\{F\}$ converges $s$-boundedly in $D\left(\sigma_{0} ; b\right)$.

From (2.8) we also find that

$$
|\Theta(X, Y)|=\left|f_{1}(X, Y ; 0)\right| \leqq M e^{b Y}\left[e^{\sigma_{\theta} X}+\left|s_{1}\right| \int_{0}^{X} e^{\sigma 0 x} d x\right] .
$$

If $\sigma_{0}>0$ or $s_{1}=0,|\Theta(X, Y)| \leqq M e^{b Y+\sigma_{0} X}\left[1+\left|s_{1}\right| / \sigma_{0}\right]=M_{1} e^{\sigma_{0} X+b Y}$. If $\sigma_{0} \leqq 0$, $s_{1} \neq 0, \mid \Theta\left(X, Y \mid \leqq M e^{b Y}\left[1+\left|s_{1}\right| X\right] \leqq M e^{b Y}\left[1+\left|s_{1}\right| / \sigma\right] e^{\sigma X}=M_{2} e^{\sigma X+b Y}\right.$ for any $\sigma>0$. Therefore, $|\Theta(X, Y)| \leqq M e^{\sigma X+\tau Y}$ in $D(c ; b)$ and we can apply Theorem 2.6 to $\Theta$.

From (1.9) with $s=0, h=s$,

$$
f_{1}(X, y ; s)=e^{-s X} \Theta(X, y)+s \int_{0}^{X} e^{-s x} \Theta(x, y) d x .
$$

For $\sigma>c$, let $\sigma_{2}$ be such that $c<\sigma_{2}<\sigma$; since $\Theta$ is of exponential type,

$$
\left|e^{-s X} \Theta(X, y)\right| \leqq M e^{-\sigma X+\sigma_{2} X+\tau y}=M e^{-\left(\sigma-\sigma_{2}\right) X+\tau y} \rightarrow 0 \text { as } X \rightarrow \infty .
$$

Therefore, $f_{1}(y ; s)=s \theta_{1}(y ; s)$. Taking the one-dimensional transform with respect to $y$, we have $f(s, t)=s \theta(s, t)$ for $\sigma>c, \tau>b$.

Corollary 1. If either $\left|f_{1}\left(X, Y ; s_{0}\right)\right| \leqq M e^{\tau_{0} Y}$ or $|\theta(X, Y)| \leqq M e^{\sigma_{0} X+r_{0} Y}$ for a given $\left(\sigma_{0}, \tau_{0}\right)$, then $L\{F\}$ converges s-boundedly in $D\left(\sigma_{0} ; \tau_{0}\right)$, and (2.10) holds and $L\{\Theta\}$ converges regularly in $D\left(c ; \tau_{0}\right)$.

If $F(x) \in \Sigma_{x}$, considered as a function of two variables $F \in \overline{\boldsymbol{\Sigma}}$. Application of Theorem 2.8 yields:

Corollary 2. Let $H(x) \in \Sigma_{x}$ and let $H_{0}(x)=\int_{0}^{x} H(u) d u$. Suppose that $\left|\int_{0}^{X} e^{-s_{1} x} H(x) d x\right| \leqq M e^{a X}$ for all $X$. Then $L_{s}\{H\}$ converges for $\sigma>\sigma_{0}=\sigma_{1}+a$; for $\sigma>c, L_{s}\left\{H_{0}\right\}$ converges regularly and

$$
h(s)=s h_{0}(s) .
$$

In particular, if either $\left|\int_{0}^{X} e^{-s_{0} x} H(x) d x\right| \leqq M$ or $\left|H_{0}(X)\right| \leqq M e^{\sigma_{0} X}$, then $L_{s}\{H\}$ converges for $\sigma>\sigma_{0}$ and, for $\sigma>c, L_{s}\left\{H_{0}\right\}$ converges regularly and (2.13) holds.

THEOREM 2.9. Let $F \in \Sigma$ and let

$$
\left|f\left(X, Y ; s_{1}, t_{1}\right)\right| \leqq M e^{a X+b Y} \quad \text { for all }(X, Y) .
$$

Set $\sigma_{0}=\sigma_{1}+a, \tau_{0}=\tau_{1}+b$ and $c=\max \left(\sigma_{0}, 0\right), d=\max \left(\tau_{0}, 0\right)$. Then $L\{F\}$ converges boundedly in $D\left(\sigma_{0} ; \tau_{0}\right), L\{\Psi\}$ converges s-boundedly in $D\left(\sigma_{0} ; d\right), L\{\Theta\}$ converges t-boundedly in $D\left(c ; \tau_{0}\right)$, and $L\{\Phi\}$ converges regularly in $D(c ; d)$. The following equalities hold in $D(c ; d)$ : 


$$
\begin{array}{rlrl}
\psi_{1}(y ; s) & =s \phi_{1}(y ; s), & \theta_{2}(x ; t) & =t \phi_{2}(x ; t), \\
\psi(s, t) & =s \phi(s, t), & \theta(s, t)=t \phi(s, t), \\
f(s, t) & =s t \phi(s, t)=s \theta(s, t)=t \psi(s, t) .
\end{array}
$$

Proof. Set $G(x, y)=f\left(x, y ; s_{1}, t_{1}\right)$. By (1.13) with $s=s_{1}, t=t_{1}, h=s-s_{1}$, $k=t-t_{1}$,

$$
\begin{aligned}
f(X, Y ; s, t)= & e^{-h X-k Y} G(X, Y)+h \int_{0}^{X} e^{-h x-k Y} G(x, Y) d x \\
& +k \int_{0}^{Y} e^{-h X-k y} G(X, y) d y \\
& +h k \int_{(0,0)}^{(X, Y)} e^{-h x-k y} G(x, y) d(x, y) .
\end{aligned}
$$

From (2.14), $\left|e^{-h x-k y} G(x, y)\right| \leqq M e^{-h_{1} x-k_{1} y}$ where $h_{1}=\sigma-\sigma_{0}, k_{1}=\tau-\tau_{0}$. When $h_{1}>0, k_{1}>0$, the first three terms on the right $\rightarrow 0$ as $(X, Y) \rightarrow \infty$ and the last term converges absolutely by the comparison test. Hence, $f(s, t)$ exists and, indeed, from (2.16)

$$
\begin{aligned}
& |f(X, Y ; s, t)| \\
& \qquad M\left[e^{-h_{1} X}+|h| \int_{0}^{X} e^{-h_{1} x} d x\right]\left[e^{-k_{1} Y}+|k| \int_{0}^{Y} e^{-k_{1} y} d y\right] .
\end{aligned}
$$

When $h_{1}>0, k_{1}>0,|f(X, Y ; s, t)| \leqq M\left[1+|h| / h_{1}\right]\left[1+|k| / k_{1}\right]=M_{1}$ whence the convergence is bounded.

Since $\int_{0}^{X} e^{-\bullet x} \Psi(x, Y) d x=f(X, Y ; s, 0)$, it follows from (2.17) that

$$
|f(X, Y ; s, 0)| \leqq M\left[e^{-h_{1} X}+|h| \int_{0}^{X} e^{-h_{1} x} d x\right]\left[e^{\tau_{0} Y}+\left|t_{1}\right| \int_{0}^{Y} e^{\tau_{0} y} d y\right] .
$$

Hence, $|f(X, Y ; s, 0)| \leqq M e^{\tau Y}$ in $D\left(\sigma_{0} ; d\right)$. By Theorem 2.8 applied to $\Psi(x, y)$, $L\{\Psi\}$ converges $s$-boundedly in $D\left(\sigma_{0} ; d\right)$. Since $\Phi(X, Y)=\int_{0}^{X} \Psi(x, Y) d x$, in $D(c ; d) L\{\Phi\}$ converges regularly and $\psi_{1}(y ; s)=s \phi_{1}(y ; s), \psi(s, t)=s \phi(s, t)$.

Similarly, $\left|\int_{0}^{Y} e^{-t y} \Theta(X, y) d y\right|=|f(X, Y ; 0, t)| \leqq M e^{\sigma X}$ in $D\left(c ; \tau_{0}\right)$. Application of the second part of Theorem 2.8 yields the $t$-bounded convergence of $L\{\Theta\}$ in $D\left(c ; \tau_{0}\right)$ and the equalities $\theta_{2}(x ; t)=t \phi_{2}(x ; t), \theta(s, t)=t \phi(s, t)$ in $D(c ; d)$.

Finally, using formula (1.13) with $s=t=0, h=s, k=t$, we find

$$
\begin{aligned}
& f(X, Y ; s, t)=e^{-s X-t Y} \Phi(X, Y)+s e^{-t Y} \int_{0}^{X} e^{-s x} \Phi(x, Y) d x \\
& +t e^{-s X} \int_{0}^{Y} e^{-t y} \Phi(X, y) d y+s t \int_{(0,0)}^{(X, Y)} e^{-s x-t y} \Phi(x, y) d(x, y) .
\end{aligned}
$$


Hence, $f(s, t)=s t \phi(s, t)$ in $D(c ; d)$.

COROLLARY. If any of the following hold:

$$
\begin{aligned}
|\Phi(X, Y)| & \leqq M e^{\sigma_{0} X+\tau_{0} Y}, & \left|f\left(X, Y ; s_{0}, t_{0}\right)\right| & \leqq M, \\
\left|\psi_{1}\left(X, Y ; s_{0}\right)\right| & \leqq M e^{\tau_{0} Y}, & \left|\theta_{2}\left(X, Y ; t_{0}\right)\right| & \leqq M e^{\sigma_{0} X},
\end{aligned}
$$

then $L\{F\}$ converges boundedly in $D\left(\sigma_{0} ; \tau_{0}\right), L\{\Psi\}$ converges s-boundedly in $D\left(\sigma_{0} ; d\right), L\{\Theta\}$ converges $t$-boundedly in $D\left(c ; \tau_{0}\right)$, and $L\{\Phi\}$ converges regularly in $D(c ; d)$. Also, $(2.15)$ holds in $D(c ; d)$.

In addition to the one-dimensional transforms $L_{s}\{F\}$ and $L_{t}\{F\}$, we shall have occasional use for "strip" transforms taken over the regions $Q_{X}$ : $0 \leqq x \leqq X, 0 \leqq y<\infty$ and $Q_{Y}: 0 \leqq x<\infty, 0 \leqq y \leqq Y$. By definition

$$
L^{X}\{F\}=\int_{Q_{X}} e^{-s x-t y} F(x, y) d(x, y)=\lim _{Y \rightarrow \infty} f(X, Y ; s, t)
$$

whenever this limit exists. We call the function so defined $f_{3}(X ; s, t)$. Since $f(X, Y ; s, t)=\int_{0}^{Y} e^{-t y} f_{1}(X, y ; s) d y$, we see that

$$
L^{x}\{F\}=L_{t}\left\{f_{1}\right\}=\int_{0}^{\infty} e^{-t y} f_{1}(X, y ; s) d y .
$$

One can also regard $L^{x}\{F\}=L\{H\}$ where $H(x, y)=F(x, y)$ in $Q_{x}$ and $H(x, y)=0$ in $Q-Q_{x}$.

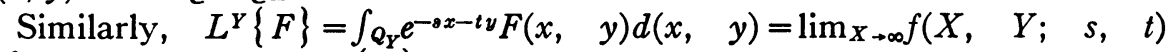
$=\int_{0}^{\infty} e^{-s x} f_{2}(x, Y ; t) d x=L_{s}\left\{f_{2}\right\}$ whenever this limit exists; we call the function so defined $f_{4}(Y ; s, t)$.

ThEOREM 2.10. Let $F \in \Sigma$.

(a) If $\left|f\left(X, Y ; s_{0}, t_{0}\right)\right| \leqq M$, then: (1) in $D\left(\sigma_{0} ; \tau_{0}\right), L^{Y}\{F\}$ converges to $f_{4}(Y ; s, t)$ for all $Y>0, L^{X}\{F\}$ converges to $f_{3}(X ; s, t)$ for all $X>0$, and

$$
f(s, t)=\lim _{X \rightarrow \infty} f_{3}(X ; s, t)=\lim _{Y \rightarrow \infty} f_{4}(Y ; s, t) .
$$

(b) If $\left|f_{1}\left(X, Y ; s_{0}\right)\right| \leqq M e^{\tau_{0} Y}$, then $L\{F\}$ converges s-boundedly in $D\left(\sigma_{0} ; \tau_{0}\right)$, (1) holds, and also: (2) $f_{4}(Y ; s, t)=\int_{0}^{Y} e^{-t y} f_{1}(y ; s) d y$ for $\sigma>\sigma_{0}$.

(c) If $\left|f_{2}\left(X, Y ; t_{0}\right)\right| \leqq M e^{\sigma_{0} X}$, then $L\{F\}$ converges $t$-boundedly in $D\left(\sigma_{0} ; \tau_{0}\right)$, (1) holds, and also: (3) $f_{3}(X ; s, t)=\int_{0}^{X} e^{-s x} f_{2}(x ; t) d x$ for $\tau>\tau_{0}$.

(d) If $|F(X, Y)| \leqq M e^{\sigma_{0} X+\tau_{0} Y}$, then $L\{F\}$ converges absolutely in $D\left(\sigma_{0} ; \tau_{0}\right)$ and (1), (2), and (3) hold.

Proof. (a) By (1.12)

$$
f\left(X, Y ; s_{0}, t\right)=e^{-k Y} f\left(X, Y ; s_{0}, t_{0}\right)+k \int_{0}^{Y} e^{-k Y} f\left(X, y ; s_{0}, t_{0}\right) d y
$$

where $k=t-t_{0}$. If $k_{1}=R(k)>0$, then 


$$
\left|f\left(X, Y ; s_{0}, t\right)\right| \leqq M\left(e^{-k_{1} Y}+|k| \int_{0}^{Y} e^{-k_{1} y} d y\right)=M\left(1+|k| / k_{1}\right)=M_{1} .
$$

On setting $h=s-s_{0}$, we obtain from (1.11)

$$
f(X, Y ; s, t)=e^{-h X_{f}} f\left(X, Y ; s_{0}, t\right)+h \int_{0}^{X} e^{-h x} f\left(x, Y ; s_{0}, t\right) d x .
$$

When $k_{1}>0$ and $h_{1}=\mathbb{R}(h)>0$, the absolute value of the first term on the right approaches 0 as $X \rightarrow \infty$ and the second term converges absolutely. Thus $f_{4}(Y ; s, t)=\lim _{X \rightarrow \infty} f(X, Y ; s, t)$ exists for $h_{1}>0, k_{1}>0 . f(s, t)$ $=\lim _{(X, Y) \rightarrow \infty} f(X, Y ; s, t)=\lim _{Y \rightarrow \infty} \lim _{X \rightarrow \infty} f(X, Y ; s, t)=\lim _{Y \rightarrow \infty} f_{4}(Y ; s, t)$.

(b) By Corollary 1 to Theorem 2.8, $L\{F\}$ converges $s$-boundedly in $D\left(\sigma_{0} ; \tau_{0}\right)$. From the first equality in (1.11)

$f(X, Y ; s, t)=e^{-h X} \int_{0}^{Y} e^{-t y} f_{1}\left(X, y ; s_{0}\right) d y+h \int_{(0,0)}^{(X, Y)} e^{-h x-t y} f_{1}\left(x, y ; s_{0}\right) d(x, y)$.

Let $h_{1}>0$.

$$
\lim _{X \rightarrow \infty} f(X, Y ; s, t)=h \int_{Q_{Y}} e^{-h x-t y} f_{1}\left(x, y ; s_{0}\right) d(x, y) .
$$

Now the integral on the right can be iterated, and so

$$
f_{4}(Y ; s, t)=h \int_{0}^{Y} e^{-t y}\left[\int_{0}^{\infty} e^{-h x} f_{1}\left(x, y ; s_{0}\right) d x\right] d y=\int_{0}^{Y} e^{-t y} f_{1}(y ; s) d y .
$$

3. Transforms of derivatives. Theorems in the previous section give the relation between the transforms of $F$ and the transforms of its integrals. By means of Lemmas 3-6 of $\$ 1$ we can use these theorems to obtain the relations between the transforms of $F$ and of its derivatives. Such relations are established under several alternative hypotheses since it is necessary sometimes to assume the existence of transforms of derivatives and at other times to assume conditions on the function itself. These results are useful in the solution of partial differential equations with constant coefficients, since transformation of the differential equation leads to an algebraic equation in two complex variables. Under more restrictive hypotheses, this application has been made by several authors.

Through this section the functions $\Phi, \Theta, \Psi, G, G_{0}, G_{1}, H, H_{0}, H_{1}, M$, $N, U, V$ will have the same meaning they had in Lemmas 3-6 of the Introduction; their transforms will be indicated by the corresponding small letters. In addition we shall use the following:

$$
\alpha(s, t)=L\left\{F_{x}\right\}, \quad \gamma(s, t)=L\left\{F_{x y}\right\}, \quad c=\max \left(\sigma_{0}, 0\right) .
$$




$$
\begin{array}{ll}
\beta(s, t)=L\left\{F_{y}\right\}, \quad & \delta(s, t)=L\left\{F_{x x}\right\}, \quad d=\max \left(\tau_{0}, 0\right), \\
\eta(s, t)=L\left\{F_{y y}\right\} . &
\end{array}
$$

We begin by giving a result for the one-dimensional transform. That it includes as special cases a number of different hypotheses employed by various authors is seen by referring to the list of subclasses of $A_{x}$ given after (1.1).

THEOREM 3.1. Let $F(x) \in \mathrm{A}_{x}$ and suppose that any one of the following holds:

(i) $|F(x)| \leqq M e^{\sigma_{0} x}$,

(ii) $L_{s}\left\{F^{\prime}\right\}$ converges at $\sigma=\sigma_{0}$,

(iii) $\left|\int_{0}^{X} e^{-s_{1} x} F^{\prime}(x) d x\right| \leqq M e^{a X}$ for all $X>0, \sigma_{0}=\sigma_{1}+a$.

Then, for $\sigma>c, L_{s}\left\{F^{\prime}\right\}$ converges to $\alpha(s), L_{s}\{F\}$ converges absolutely, $F$ is of exponential type, and

$$
\alpha(s)=s f(s)-F(+0), \quad \sigma>c .
$$

Proof. Let (iii) hold. Since $F^{\prime}(x) \in \Sigma_{x}$, Corollary 2 of Theorem 2.8 can be applied to $F^{\prime}(x)$ instead of $H(x)$; then $\Theta_{1}(x)=\int_{0}^{x} F^{\prime}(u) d u=F(x)-F(+0)$ plays the role of $H_{0}(x)$. For $\sigma>\sigma_{0}, L_{s}\left\{F^{\prime}\right\}$ converges; for $\sigma>c, L_{s}\left\{\Theta_{1}\right\}$ converges absolutely and $\alpha(s)=s \theta_{1}(s)$. But for $\sigma>0, L_{s}\{F(+0)\}=F(+0) / s$ so that when $\sigma>c, L_{\mathbf{s}}\{F\}$ converges absolutely and $f(s)-F(+0) / s=\theta_{1}(s)$. Substituting in the formula for $\alpha(s)$, we get (3.1). Indeed, $|F(x)| \leqq|F(+0)|$ $+\left|\Theta_{1}(x)\right| \leqq M_{1} e^{\sigma x}$ for $\sigma>c$.

If (ii) holds, then $\left|\int_{0}^{X} e^{-s 0 x} F^{\prime}(x) d x\right| \leqq M$ for all $X>0$, and so we have a special case of (iii) with $a=0$.

If (i) holds, $\left|\int_{0}^{X} F^{\prime}(x) d x\right|=|F(X)-F(+0)| \leqq M\left[e^{\sigma_{0} X}+1\right] \leqq 2 M e^{c x}$. This is a special case of (iii) with $s_{1}=0, a=c$.

Theorem 3.2. Let $F(x, y) \in A_{x}$ for $y>0, G(y) \in \Sigma_{y}, F_{x} \in \Sigma$. Let any one of the following hold:

(i) $L\{F\}$ converges t-boundedly at $\left(s_{0}, t_{0}\right)$,

(ii) $L\left\{F_{x}\right\}$ converges boundedly and $L_{t}\{G\}$ converges at $\left(s_{0}, t_{0}\right)$,

(iii) $\left|\int_{(0,0)}^{(X),} e^{-s_{1} x-t_{1} y} F_{x}(x, y) d(x, y)\right| \leqq M e^{a X+b Y},\left|\int_{0}^{Y} e^{-t_{1} y} G(y) d y\right| \leqq M e^{b Y}$, and $\sigma_{0}=\sigma_{1}+a, \tau_{0}=\tau_{1}+b$.

Then $L\left\{F_{x}\right\}$ converges boundedly to $\alpha(s, t)$ in $D\left(\sigma_{0} ; \tau_{0}\right), L_{t}\{G\}$ converges for $\tau>\tau_{0} ; L\{F\}$ and $L\{\Theta\}$ converge t-boundedly in $D\left(c ; \tau_{0}\right) ; L\{M\}$ converges s-boundedly in $D\left(\sigma_{0} ; d\right) ; L\{\Psi\}$ and $L\{\Phi\}$ converge regularly in $D(c ; d)$; $L_{t}\left\{G_{0}\right\}$ converges regularly for $\tau>d$.

Formulas (2.12) and (2.15) hold and also in $D(c ; d)$

$$
\begin{aligned}
\alpha(s, t) & =s f(s, t)-g(t)=t m(s, t)=s t \psi(s, t)-g(t), \\
m(s, t) & =s \psi(s, t)-g(t) / t, \\
m_{1}(y ; s) & =s \psi_{1}(y ; s)-G_{0}(y) .
\end{aligned}
$$


Proof. Assume first that (iii) holds. By Corollary 2 to Theorem 2.8, $L_{t}\{G\}$ converges for $\tau>\tau_{0}$ and $G_{0}$ is of exponential type for $\tau>d$. Hence, $L\{G\}$ converges $t$-boundedly to $g(t) / s$ in $D\left(0 ; \tau_{0}\right)$ and $L\left\{G_{0}\right\}$ converges regularly to $g(t) / s t$ in $D(0 ; d)$. Apply Theorem 2.9 to $F_{x}$ instead of $F$ whence $L\left\{F_{x}\right\}$ converges boundedly in $D\left(\sigma_{0} ; \tau_{0}\right), L\{M\}$ converges $s$-boundedly in $D\left(\sigma_{0} ; d\right), L\{P\}$ converges $t$-boundedly in $D\left(c ; \tau_{0}\right)$, and $L\{Q\}$ converges regularly in $D(c ; d)$ (where $P(x, y)=\int_{0}^{x} F_{x}(u, y) d u$ and $Q(x, y)=\int_{(0,0)}^{(x, y)} F_{x}(u, v) d(u, v)$ ). By (1.14), $F=P+G$ so that $L\{F\}$ converges $t$-boundedly in $D\left(c ; \tau_{0}\right)$ to $f(s, t)=p(s, t)+g(t) / s$; by $(1.15) \Psi=Q+G_{0}$ so that $L\{\Psi\}$ converges regularly in $D(c ; d)$ to $\psi(s, t)=q(s, t)+g(t) / s t$. Making the necessary adjustments in formulas (2.15) and substituting, we have (2.12) and (3.2) in $D(c ; d)$. Since $L\{F\}$ converges boundedly in $D\left(c ; \tau_{0}\right)$, by the Corollary to Theorem 2.9 $L\{\Theta\}$ converges $t$-boundedly in $D\left(c ; \tau_{0}\right)$ and $L\{\Phi\}$ converges regularly in $D(c ; d)$ and formulas (2.15) hold.

Hypothesis (ii) is a special case of (iii) with $a=b=0$.

We know that $\int_{0}^{Y} e^{-t_{0} y} G(y) d y=\lim _{x \rightarrow 0+} \int_{0}^{Y} e^{-t_{0} y} F(x, y) d y$ on applying Lemma 3 to $e^{-t_{0} y} F(x, y)$ which is also a function of class $\mathrm{A}_{x}$. Under hypothesis (i) then, $\left|\int_{0}^{Y} e^{-t_{0} y} G(y) d y\right|=\lim _{x \rightarrow 0+}\left|f_{2}\left(x, Y ; t_{0}\right)\right| \leqq \lim _{x \rightarrow 0+} M e^{\sigma_{0} x}=M$ and, therefore, $\quad\left|\int_{(0,0)}^{(X, Y)} e^{-s x-t_{0} y} F_{x}(x, \quad y) d(x, \quad y)\right|=\mid \int_{0}^{Y} e^{-t_{0} y}\left[e^{-s X} F(X, \quad y)-G(y)\right.$ $\left.+s \int_{0}^{X} e^{-s x} F(x, y) d x\right] d y\left|\leqq M e^{-\sigma X+\sigma_{0} X}+M+M\right| s \mid \int_{0}^{X} e^{-\sigma x+\sigma_{0} x} d x \leqq M_{1}$ for all $(X, Y)$ and $\sigma>\sigma_{0}$. Thus hypothesis (iii) holds with $s_{1}=\bar{s}, \bar{\sigma}>\sigma_{0}, \tau_{1}=\tau_{0}$, $a=b=0$.

A parallel result holds concerning $L\left\{F_{y}\right\}=\beta(s, t)$. Instead of (2.12), (2.15) and (3.2) we have in $D(c ; d)(2.10),(2.15)$ and

$$
\begin{aligned}
\beta(s, t) & =t f(s, t)-h(s)=s n(s, t)=s t \theta(s, t)-h(s), \\
n(s, t) & =t \theta(s, t)-h(s) / s, \\
n_{2}(x ; t) & =t \theta_{2}(x ; t)-H_{0}(x) .
\end{aligned}
$$

We have the following result concerning both first derivatives:

THEOREM 3.3. Let $F(x, y) \in A^{*}$ and let any one of the following hold:

(i) $L\{F\}$ converges s-boundedly and $t$-boundedly at $\left(s_{0}, t_{0}\right)$,

(ii) $L\left\{F_{x}\right\}$ and $L\left\{F_{y}\right\}$ converge boundedly at $\left(s_{0}, t_{0}\right)$,

(iii)

$$
\begin{aligned}
& \left|\int_{(0,0)}^{(X, Y)} e^{-\varepsilon_{1} x-t_{1} y} F_{x}(x, y) d(x, y)\right| \leqq M e^{a X+b Y}, \\
& \left|\int_{(0,0)}^{(X, Y)} e^{-\varepsilon_{1} x-t_{1} y} F_{y}(x, y) d(x, y)\right| \leqq M e^{a X+b Y},
\end{aligned}
$$

and $\sigma_{0}=\sigma_{1}+a, \tau_{0}=\tau_{1}+b$.

Then in $D(c ; d), L\left\{F_{x}\right\}, L\left\{F_{y}\right\}, L_{t}\{G\}, L_{s}\{H\}$ converge boundedly, $L\{F\}$ converges s-boundedly and t-boundedly, $L\{M\}$ converges s-boundedly, $L\{N\}$ 
converges t-boundedly, and $L\{\Theta\}, L\{\Psi\}, L\{\Phi\}, L_{8}\left\{H_{0}\right\}, L_{t}\left\{G_{0}\right\}$ converge regularly. Formulas (2.10), (2.12), (2.15), (3.2), and (3.3) hold.

Proof. By Lemma 3(c), formulas (1.14) and (1.16) hold, so that

$$
G(y)=H(x)+\int_{0}^{y} F_{y}(x, v) d v-\int_{0}^{x} F_{x}(u, y) d u .
$$

When (iii) is true and $(s, t)$ is a point in $D(c ; d)$, Theorem 2.9 applied to $F_{x}$ and to $F_{y}$ gives the bounded convergence of the double transforms of the two integrals on the right side of the above formula. Hence, on multiplying this equation by $e^{-s x-t y}$ and integrating over the rectangle $R_{B Y}$ where $B$ is a fixed value of $X>0$, it follows that for all $Y>0$,

$$
\begin{aligned}
\frac{1}{|s|}\left|1-e^{-s B}\right| \cdot\left|\int_{0}^{y} e^{-t y} G(y) d y\right| & \\
& \leqq \frac{1}{|t|}\left[1-e^{-t y}\right] \cdot \mid \int_{0}^{B} e^{-s x} H(x) d x+M_{1}+M_{2} .
\end{aligned}
$$

Thus

$$
\left|\int_{0}^{Y} e^{-t y} G(y) d y\right| \leqq M, \quad \text { for all } Y>0 .
$$

By Corollary 2 to Theorem 2.8, $L_{t}\{G\}$ converges for $\tau>d$. For any $(s, t)$ $\in D(c ; d)$ there exist $s_{2}$ and $t_{2}$ with $c<\sigma_{2}<\sigma, d<\tau_{2}<\tau$ such that $L\left\{F_{x}\right\}$ converges boundedly and $L_{t}\{G\}$ converges at $\left(s_{2}, t_{2}\right)$. Apply part (ii) of Theorem 3.2. Similarly, $L_{s}\{H\}$ converges $(\sigma>c)$ whence the parallel theorem may be applied.

Hypotheses (ii) and (i) lead to (iii) just as in the previous theorem.

For the second derivatives we give the region as $D(c ; d)$ for simplicity. It is evident that if $F_{x x}$ satisfies the hypotheses on $F_{x}$ given in. Theorem 3.2 we have the following result from Lemma 5:

Theorem 3.4. Let $F_{x}(x, y) \in \mathrm{A}_{x}$ for $y>0, F_{x x} \in \Sigma, G_{1}(y)=F_{x}(+0, y) \in \Sigma_{y}$, $G(y) \in \Sigma_{y}$. Let any one of the following hold:

(i) $L\left\{F_{x}\right\}$ converges $t$-boundedly and $L_{t}\{G\}$ converges at $\left(s_{0}, t_{0}\right)$,

(ii) $L\left\{F_{x x}\right\}$ converges boundedly, $L_{t}\{G\}$ and $L_{t}\left\{G_{1}\right\}$ converge at $\left(s_{0}, t_{0}\right)$,

(iii)

$$
\begin{gathered}
\left|\int_{(0,0)}^{(X, Y)} e^{-s_{1} x-t_{1} y} F_{x x}(x, y) d(x, y)\right| \leqq M e^{a X+b Y}, \\
\left|\int_{0}^{Y} e^{-t_{1} y} G_{1}(y) d y\right| \leqq M e^{b Y}, \quad\left|\int_{0}^{Y} e^{-t_{1} y} G(y) d y\right| \leqq M e^{b Y},
\end{gathered}
$$

and $\sigma_{0}=\sigma_{1}+a, \tau_{0}=\tau_{1}+b$. 
Then in $D(c ; d), L\left\{F_{x x}\right\}$ converges boundedly to $\delta(s, t), L_{t}\{G\}$ and $L_{t}\left\{G_{1}\right\}$ converge, $L\left\{F_{x}\right\}, L\{F\}, L\{\Theta\}$ converge t-boundedly, $L\{V\}$ converges s-boundedly, $L\{M\}, L_{t}\left\{G_{0}\right\}, L\{\Psi\}, L\{\Phi\}$ converge regularly. Formulas (2.12), (2.15), (3.2) hold and in addition:

$$
\delta(s, t)=t v(s, t)=s \alpha(s, t)-g_{1}(t)=s^{2} f(s, t)-s g(t)-g_{1}(t),
$$

$$
\begin{aligned}
\alpha_{2}(x ; t) & =t m_{2}(x ; t), \quad v_{1}(y ; s)=s m_{1}(y ; s)-\int_{0}^{y} G_{1}(z) d z, \\
v(s, t) & =s m(s, t)-g_{1}(t) / t .
\end{aligned}
$$

A parallel theorem holds regarding $L\left\{F_{y y}\right\}=\eta(s, t)$. The formulas corresponding to (3.4) are

$$
\eta(s, t)=s u(s, t)=t \beta(s, t)-h_{2}(s)=t^{2} f(s, t)-t h(s)-h_{2}(s),
$$

$$
\begin{aligned}
\beta_{1}(y ; s) & =\operatorname{sn}_{1}(y ; s), \quad u_{2}(x ; t)=\operatorname{tn}_{2}(x ; t)-\int_{0}^{x} H_{2}(z) d z, \\
u(s, t) & =\operatorname{tn}(s, t)-h_{2}(s) / s .
\end{aligned}
$$

For the cross-partial $F_{x y}$ we have the following theorem when $F \in A$, using Lemma 4:

Theorem 3.5. Let $F(x, y) \in A$. Assume that any one of the following holds:

(i) $|F(x, y)| \leqq M e^{\sigma_{0} x+r_{0} y}$,

(ii') $L\left\{F_{y}\right\}$ converges $t$-boundedly at $\left(s_{0}, t_{0}\right)$ and $L_{s}\left\{H^{\prime}\right\}$ converges at $s_{0}$ or $|H(X)| \leqq M e^{\sigma_{0} x}$,

(ii) $L\left\{F_{x}\right\}$ converges s-boundedly at $\left(s_{0}, t_{0}\right)$ and $L_{t}\left\{G^{\prime}\right\}$ converges at $t_{0}$ or $|G(Y)| \leqq M e^{r_{0} Y}$,

(iii') $L\left\{F_{x y}\right\}$ converges boundedly at $\left(s_{0}, t_{0}\right), L_{t}\left\{G^{\prime}\right\}$ converges at $t_{0}$, and $L_{s}\left\{H^{\prime}\right\}$ converges at $s_{0}$,

(iii)

$$
\begin{gathered}
\left|\int_{(0,0)}^{(X, Y)} e^{-s 1 x-t_{1} y} F_{x y}(x, y) d(x, y)\right| \leqq M e^{a X+b Y}, \\
\left|\int_{0}^{Y} e^{-t_{1} y} G^{\prime}(y) d y\right| \leqq M e^{b Y}, \quad\left|\int_{0}^{X} e^{-s_{1} x} H^{\prime}(x) d x\right| \leqq M e^{a X},
\end{gathered}
$$

and $\sigma_{0}=\sigma_{1}+a, \tau_{0}=\tau_{1}+b$.

Then in $D(c ; d), L\left\{F_{x y}\right\}$ converges boundedly to $\gamma(s, t), L\left\{F_{y}\right\}$ and $L\{N\}$ converge t-boundedly, $L\left\{F_{x}\right\}$ and $L\{M\}$ converge s-boundedly, $L\{F\}, L\{\Theta\}$, $L\{\Psi\}, L\{\Phi\}, L_{s}\{H\}$, and $L_{t}\{G\}$ converge regularly, $L_{s}\left\{H^{\prime}\right\}$ and $L_{t}\left\{G^{\prime}\right\}$ converge. Formulas (2.10), (2.12), (2.15), (3.2), (3.3) hold and

$$
\begin{aligned}
\gamma(s, t) & =s \beta(s, t)-t g(t)+F(+0,+0)=t \alpha(s, t)-s h(s)+F(+0,+0) \\
& =s t f(s, t)-s h(s)-t g(t)+F(+0,+0) \\
\alpha_{1}(y ; s) & =s f_{1}(y ; s)-G(y) ; \quad \beta_{2}(x ; t)=t f_{2}(x ; t)-H(x) .
\end{aligned}
$$


Proof. Under hypothesis (iii) we know from Theorem 2.9 that, in $D(c ; d)$, $L\left\{F_{x y}\right\}$ converges boundedly, $L\{R\}$ converges $t$-boundedly, $L\{W\}$ converges $s$-boundedly, and $L\{Z\}$ converges regularly where, by Lemma 4 , $R(x, y)=\int_{0}^{x} \overline{F_{x y}}(u, y) d u=F_{y}(x, y)-G^{\prime}(y)$ p.p. on $J, W(x, y)=\int_{0}^{y} \overline{F_{x y}}(x, v) d v$ $=F_{x}(x, y)-H^{\prime}(x)$ p.p. on $I$, and $Z(x, y)=\int_{(0,0)}^{(x, y)} \overline{F_{x y}}(u, v) d(u, v)=F-G-H+c_{0}$. But $L_{t}\left\{G^{\prime}\right\}$ converges for $\tau>\tau_{0}$ and $L_{\mathbf{s}}\left\{H^{\prime}\right\}$ converges for $\sigma>\sigma_{0}$, whence $L\left\{G^{\prime}\right\}$ converges $t$-boundedly, $L\{G\}$ converges regularly, $L\left\{H^{\prime}\right\}$ converges $s$-boundedly, and $L\{H\}$ converges regularly in $D(c ; d)$ by Theorem 3.1. Therefore $L\left\{F_{y}\right\}$ converges $t$-boundedly, $L\left\{F_{x}\right\}$ converges $s$-boundedly, and $L\{F\}$ converges regularly. Substitution in (2.15) yields (3.6). By Lemma 4, $F \in \mathrm{A}^{*}$ and the other hypotheses of Theorem 3.3 hold, and so the remaining results follow.

Now when hypothesis (i) holds, $|G(y)| \leqq M e^{\tau_{0} y},|H(x)| \leqq M e^{\sigma_{0} x},\left|c_{0}\right| \leqq M$, and hence $|Z| \leqq 4 M e^{c x+d y},\left|\int_{0}^{Y} G^{\prime}(y) d y\right| \leqq 2 M e^{d Y},\left|\int_{0}^{X} H^{\prime}(x) d x\right| \leqq 2 M e^{c X}$. Thus we have (iii) with $s_{1}=t_{1}=0$ and $a=c, b=d$. When (iii') holds, (iii) is true with $a=b=0$.

When hypothesis (ii') holds, $\left|\int_{0}^{Y} F_{y}(x, v) d v\right| \leqq M e^{\sigma X+\tau Y}$ by Theorem 2.8. Since $F(x, Y)=H(x)+\int_{0}^{Y} F_{y}(x, v) d v,|F(X, Y)| \leqq 2 M e^{\sigma X+\tau Y}$ in $D(c ; d)$, which means that we can proceed as under hypothesis (i). A similar statement is true when (ii) holds.

Finally we have:

TheOREM 3. 6. Let $F_{x}$ and $F_{y}$ be in class $\mathrm{A}^{*}$ and let any one of the following hold:

(i) $L\left\{F_{x}\right\}$ and $L\left\{F_{y}\right\}$ converge s-boundedly and t-boundedly at $\left(s_{0}, t_{0}\right)$,

(ii) $L\left\{F_{x x}\right\}, L\left\{F_{x y}\right\}, L\left\{F_{y y}\right\}$ converge boundedly at $\left(s_{0}, t_{0}\right)$,

(iii)

$$
\begin{aligned}
& \left|\int_{(0,0)}^{(X, Y)} e^{-s_{1} x-t_{1} y} F_{x x}(x, y) d(x, y)\right| \leqq M e^{a X+b Y}, \\
& \left|\int_{(0,0)}^{(X, Y)} e^{-s_{1} x-t_{1} y} F_{x y}(x, y) d(x, y)\right| \leqq M e^{a X+b Y}, \\
& \left|\int_{(0,0)}^{(X, Y)} e^{-s_{1} x-t_{1} y} F_{y y}(x, y) d(x, y)\right| \leqq M e^{a X+b Y},
\end{aligned}
$$

and $\sigma_{0}=\sigma_{1}+a, \tau_{0}=\tau_{1}+b$.

Then in $D(c ; d), L\left\{F_{x x}\right\}, L\left\{F_{x y}\right\}, L\left\{F_{y y}\right\}, L_{s}\left\{H_{2}\right\}, L_{s}\left\{H^{\prime}\right\}, L_{t}\left\{G_{1}\right\}$, $L_{t}\left\{G^{\prime}\right\}$ converge boundedly, $L\left\{F_{x}\right\}, L\left\{F_{y}\right\}, L\{V\}$ converge s-boundedly, $L\left\{F_{x}\right\}, L\left\{F_{y}\right\}, L\{U\}$ converge t-boundedly, $L\{F\}, L\{M\}, L\{N\}, L_{t}\{G\}$, $L_{s}\{H\}, L\{\Theta\}, L\{\Psi\}, L\{\Phi\}$ converge regularly. Formulas (2.10), (2.12), (2.15), (3.2)-(3.6) hold.

Proof. Assume (iii) holds. From Lemma $6, F$ is in class A and, hence, $G_{1}$ and $H_{2}$ are in $\Sigma$ and $G^{\prime}(y)=F_{y}(+0, y)$ p.p., $H^{\prime}(x)=F_{x}(x,+0)$ p.p. From Theorem 3.3 applied to $F_{x}$ and $F_{y}$ separately, we find that $L\left\{F_{x x}\right\}, L\left\{F_{x y}\right\}$, 
$L\left\{F_{y y}\right\}$ converge boundedly, $L\left\{F_{x}\right\}$ and $L\left\{F_{y}\right\}$ converge $s$-boundedly and $t$-boundedly, $L\{V\}$ converges $s$-boundedly, $L\{U\}$ converges $t$-boundedly, $L\{M\}$ and $L\{N\}$ converge regularly, and $L_{t}\left\{G_{1}\right\}, L_{t}\left\{G^{\prime}\right\}, L_{s}\left\{H_{2}\right\}, L_{s}\left\{H^{\prime}\right\}$ converge. But then hypothesis (ii) of Theorem 3.5 holds and thus from Theorem 3.5 we obtain the regular convergence of $L\{F\}, L\{\Theta\}, L\{\Psi\}, L\{\Phi\}$ and the corresponding formulas.

If (ii) holds, (iii) is true with $a=b=0$.

If (i) holds, apply Theorem 3.3 (i) to $F_{x}$ and $F_{y}$ to show that (iii) holds with $a=b=0$ and $\left(s_{1}, t_{1}\right) \in D(c ; d)$.

Theorems 3.3 and 3.6 are most useful in general first and second order partial differential equations. Indeed, hypotheses there can of ten be combined with hypotheses that $F$ or $F_{x}$ and $F_{y}$ be in class $\mathrm{A}^{*}$.

4. Linear transformations. Let $L_{1}$ and $L_{2}$ be two rays emanating from $P_{0}\left(x_{0}, y_{0}\right)$ and having the parametric representation

$$
\begin{aligned}
& L_{1}: \quad x=x_{0}+a \alpha_{1}, \quad L_{2}: \quad x=x_{0}+c \alpha_{2}, \\
& y=y_{0}+b \alpha_{1}, \quad y=y_{0}+d \alpha_{2}, \\
& \left(0 \leqq \alpha_{i}<\infty\right)
\end{aligned}
$$

where $\Delta=a d-b c \neq 0$. By the sector $S^{0}$ between $L_{1}$ and $L_{2}$ we mean the open set of points $P$ such that parallels to $L_{1}$ and $L_{2}$ through $P$ intersect both $L_{2}$ and $L_{1}$ at finite points $P_{i}$. If the coordinates of $P_{i}$ given by (4.1) correspond to certain values $\alpha_{i}$ of the parameters, then the coordinates of $P$ are

$$
x=x_{0}+a \alpha_{1}+c \alpha_{2}, \quad y=y_{0}+b \alpha_{1}+d \alpha_{2} .
$$

Conversely any point whose coordinates are given by (4.2) for some $\alpha_{1}>0$, $\alpha_{2}>0$ lies in $S^{0}$. On solving (4.2) for $\alpha_{1}$ and $\alpha_{2}$, we see that $S^{0}$ may also be considered as the set of all points $(x, y)$ such that

$$
\left[d\left(x-x_{0}\right)-c\left(y-y_{0}\right)\right] / \Delta>0,\left[-b\left(x-x_{0}\right)+a\left(y-y_{0}\right)\right] / \Delta>0 .
$$

The closure of $S^{0}, S=S^{0}+L_{1}+L_{2}$, is the set of all points whose coordinates are of the form (4.2) where $\alpha_{1} \geqq 0, \alpha_{2} \geqq 0$.

Under the linear transformation

$$
\begin{aligned}
T: x & =x_{0}+a x^{\prime}+c y^{\prime} \\
y & =y_{0}+b x^{\prime}+d y^{\prime}
\end{aligned}
$$

with inverse

$$
\begin{aligned}
T^{-1}: & x^{\prime}=\left[d\left(x-x_{0}\right)-c\left(y-y_{0}\right)\right] / \Delta, \\
y^{\prime} & =\left[-b\left(x-x_{0}\right)+a\left(y-y_{0}\right)\right] / \Delta
\end{aligned}
$$

$P_{0} \rightarrow(0,0), L_{1} \rightarrow L_{1}^{\prime}: x^{\prime}=\alpha_{1}, y^{\prime}=0 \quad\left(0 \leqq \alpha_{1}\right)$ the positive half of the $x$-axis, $L_{2} \rightarrow L_{2}^{\prime}: x^{\prime}=0, y^{\prime}=\alpha_{2}\left(0 \leqq \alpha_{2}\right)$ the positive half of the $y$-axis, $S^{0} \rightarrow x^{\prime}=\alpha_{1}$, $y^{\prime}=\alpha_{2}\left(0<\alpha_{i}\right)$ the first quadrant $Q^{0}$. A parallelogram $S_{X Y}$ consisting of points (4.2), where $0 \leqq \alpha_{1} \leqq X, 0 \leqq \alpha_{2} \leqq Y$, is transformed into a rectangle $R_{X Y}: 0 \leqq x^{\prime}$ $\leqq X, 0 \leqq y^{\prime} \leqq Y$. 
If $G(x, y)$ is defined on a measurable kernel $W$ of $S$, the function $H\left(x^{\prime}, y^{\prime}\right)$ $=G\left(x_{0}+a x^{\prime}+c y^{\prime}, y_{0}+b x^{\prime}+d y^{\prime}\right)$ is defined on a measurable kernel of $Q$. When $H \in \Sigma$, its extended function $H_{0}$ is summable in every $R_{X Y}$; hence, if we let $G_{0}$ be the function equal to $G$ on $W$ and equal to 0 on $S-W$, it is summable in the transformed region $S_{X Y}$ and( $\left.{ }^{10}\right)$

$$
\int_{S_{X Y}} G_{0}(x, y) d(x, y)=|\Delta| \int_{R_{X Y}} H_{0}\left(x^{\prime}, y^{\prime}\right) d\left(x^{\prime}, y^{\prime}\right) .
$$

We define

$$
\int_{S} G(x, y) d(x, y)=|\Delta| \int_{Q} H\left(x^{\prime}, y^{\prime}\right) d\left(x^{\prime}, y^{\prime}\right)
$$

whenever the integral on the right exists. It follows from (4.5) that $\int_{S} G(x, y) d(x, y)$ exists if and only if $\lim _{X, Y \rightarrow \infty} \int_{S_{X Y}} G(x, y) d(x, y)$ exists and is equal to it.

THEOREM 4.1. Let $a, b, c, d, x_{0}$ and $y_{0}$ be real constants with $\Delta=a d-b c \neq 0$ and let $p$ and $q$ be complex. Let $F \in \Sigma$ and define

$$
\begin{gathered}
V(x, y)=\frac{1}{|\Delta|} F\left\{\frac{d\left(x-x_{0}\right)-c\left(y-y_{0}\right)}{\Delta}, \frac{-b\left(x-x_{0}\right)+a\left(y-y_{0}\right)}{\Delta}\right\} \\
\cdot \exp \left(-p\left\{\frac{d\left(x-x_{0}\right)-c\left(y-y_{0}\right)}{\Delta}\right\}\right. \\
\left.-q\left\{\frac{-b\left(x-x_{0}\right)+a\left(y-y_{0}\right)}{\Delta}\right\}\right)
\end{gathered}
$$

in the sector $S$ defined in (4.3). If $L\{F\}$ converges boundedly for $(s, t) \in D$, then $\int_{s} e^{-s x-t y} V(x, y) d(x, y)$ converges for all $(s, t)$ such that $(a s+b t+p$, cs $+d t+q) \in D$ and

$$
e^{-s x_{0}-t y_{0}} f(a s+b t+p, c s+d t+q)=\int_{S} e^{-s x-t y} V(x, y) d(x, y) .
$$

Furthermore,

$$
\left|\int_{S_{X Y}} e^{-s x-t y} V(x, y) d(x, y)\right| \leqq M \quad \text { for all } X>0, Y>0 .
$$

Proof. Under the transformation $T$ given in (4.4), $S \rightarrow Q$,

$$
\begin{aligned}
& e^{-s x-t y} V(x, y) \rightarrow H\left(x^{\prime}, y^{\prime}\right) \\
& \quad=(1 /|\Delta|) F\left(x^{\prime}, y^{\prime}\right) \exp \left(-s x_{0}-t y_{0}-(a s+b t+p) x^{\prime}-(c s+d t+q) y^{\prime}\right) .
\end{aligned}
$$

(10) E. W. Hobson, Theory of functions of a real variable, 3d Ed., Cambridge University Press, 1927, vol. 1, p. 653. 
Whenever $(a s+b t+p, c s+d t+q) \in D, \int_{Q} H\left(x^{\prime}, y^{\prime}\right) d\left(x^{\prime}, y^{\prime}\right)$ exists. Hence, by (4.6), $\int_{S} e^{-s x-t y} V(x, y) d(x, y)$ exists and (4.8) holds.

THEOREM 4.2. Let the hypotheses of Theorem 4.1 hold and assume further that

$$
\int_{Q-R} \exp \left(-(a s+b t+p) x^{\prime}-(c s+d t+q) y^{\prime}\right) F\left(x^{\prime}, y^{\prime}\right) d\left(x^{\prime}, y^{\prime}\right)=0
$$

where

$$
R: \quad x_{0}+a x^{\prime}+c y^{\prime} \geqq 0, \quad y_{0}+b x^{\prime}+d y^{\prime} \geqq 0 .
$$

Define $V(x, y)$ as in (4.7) and

$$
W(x, y)=V(x, y) \text { in } S Q ; \quad W(x, y)=0 \text { in } Q-S .
$$

Then $L\{W\}$ converges boundedly for all $(s, t)$ such that $(a s+b t+p, c s+d t+q)$ $\in D$ and

$$
w(s, t)=e^{-s x_{0}-t y_{0}} f(a s+b t+p, c s+d t+q) .
$$

Proof. Under the transformation $T, S \rightarrow Q$ and $Q \rightarrow R$ and $e^{-s x-t y} V(x, y) \rightarrow$ $H\left(x^{\prime}, y^{\prime}\right)$ defined in the preceding proof. Hence, $\int_{s-e^{-s x-t y}} e^{-s}(x, y) d(x, y)=0$ by (4.10). Therefore, for $(a s+b t+p, c s+d t+q) \in D$ by (4.8)

$$
\begin{aligned}
e^{-s x_{0}-t y_{0}} f(a s+b t+p, c s+d t+q) & =\int_{S} e^{-s x-t y} V(x, y) d(x, y) \\
& =\int_{S Q} e^{-s x-t y} V(x, y) d(x, y) \\
& =\int_{Q} e^{-s x-t y} W(x, y) d(x, y) .
\end{aligned}
$$

The preceding theorem gives a condition undęr which an expression of the form $e^{-s x_{0}-t y} 0 f(a s+b t+p, c s+d t+q)$ is a transform. The following corollaries give important special cases of this:

Corollary 1 (Real Translation). Let $L\{F\}$ converge boundedly in $D$ and let

$$
F(x, y)=0 \text { p.p. in } Q-R \text { where } R: x \geqq-x_{0}, y \geqq-y_{0} .
$$

Set $W(x, y)=F\left(x-x_{0}, y-y_{0}\right)$ in $S Q, W(x, y)=0$ in $Q-S$ where $S: x \geqq x_{0}$, $y \geqq y_{0}$. Then $L\{W\}$ converges boundedly in $D$ and

$$
w(s, t)=e^{-s x_{0}-t y_{0}} f(s, t) .
$$

If $x_{0} \geqq 0, y_{0} \geqq 0$, condition (4.12) is vacuously true.

Proof. Apply the theorem with $a=d=1, b=c=p=q=0$. 
Corollary 2 (Scale change). Given $a>0, d>0$, define

$$
W(x, y)=\frac{1}{a d} F\left(\frac{x}{a}, \frac{y}{d}\right)
$$

in $Q$. Then for all $(s, t)$ such that $(a s, d t)$ is in the region of bounded convergence of $L\{F\}, L\{W\}$ converges boundedly and

$$
w(s, t)=f(a s, d t) .
$$

Corollary 3 (Complex translation). If $p$ and $q$ are complex, and if

$$
W(x, y)=e^{-p x-q y} F(x, y)
$$

in $Q$, then for all $(s, t)$ such that $(s+p, t+q)$ is within the region of bounded convergence of $L\{F\}, L\{W\}$ converges boundedly and

$$
w(s, t)=f(s+p, t+q) .
$$

CoRollary 4 (COMPleX linear TRANSFormation). Let $a, b, c, d$ be real constants with $\Delta=a d-b c \neq 0$ and $p$ and $q$ complex. Let $S:(d x-c y) / \Delta \geqq 0$, $(-b x+a y) / \Delta \geqq 0$ and $R: a x+c y \geqq 0, b x+d y \geqq 0$. Set

$$
\begin{array}{rlr}
W(x, y)= & \frac{1}{|\Delta|} F\left(\frac{d x-c y}{\Delta}, \frac{-b x+a y}{\Delta}\right) \\
& \cdot \exp \left(-p\left(\frac{d x-c y}{\Delta}\right)-q\left(\frac{-b x+a y}{\Delta}\right)\right) & \text { in } S Q, \\
W(x, y)= & 0 \quad \text { in } Q-S,
\end{array}
$$

and assume that

$$
F(x, y)=0 \text { p.p. in } Q-R .
$$

Then if $L\{F\}$ converges boundedly in $D, L\{W\}$ converges boundedly for all $(s, t)$ such that $(a s+b t+p, c s+d t+q) \in D$ and

$$
w(s, t)=f(a s+b t+p, c s+d t+q) .
$$

When $a, b, c, d$ are non-negative, $Q \subseteq R$, (4.19) is vacuously true, and $S Q=S$.

When $a / \Delta,-b / \Delta,-c / \Delta, d / \Delta$ are non-negative, $S Q=Q$ and $R \subseteq Q$.

Theorem 4.3. Let $b>0, d>0, p$ and $q$ complex, and let $L\{F\}$ converge boundedly in $D\left(\sigma_{0} ; \tau_{0}\right)$. On setting

$$
U(y)=\frac{1}{d} \int_{0}^{y / b} F\left(x, \frac{y-b x}{d}\right) \exp \left(-p x-q\left(\frac{y-b x}{d}\right)\right) d x,
$$

$L_{t}\{U\}$ converges for all $\tau>\max \left[\left(\tau_{0}-R(q)\right) / d,\left(\sigma_{0}-R(p)\right) / b\right]=\tau_{1}$ and 


$$
u(t)=f(b t+p, d t+q) .
$$

Proof. Applying Corollary 4 with $a=1, c=0$, we find that $\Delta=d>0$ and (4.19) is vacuously true. The function

$$
\begin{array}{lrl}
W(x, y) & =\frac{1}{d} F\left(x, \frac{y-b x}{d}\right) \exp \left(-p x-q\left(\frac{y-b x}{d}\right)\right) & \text { in } S: y \geqq b x \geqq 0, \\
W(x, y) & =0 & \text { in } Q-S: 0 \leqq y<b x,
\end{array}
$$

has a double transform for all $(s, t)$ such that $(s+b t+p, d t+q) \in D\left(\sigma_{0} ; \tau_{0}\right)$ and $w(s, t)=f(s+b t+p, d t+q)$. Then $L\{W\}$ converges boundedly in $D\left(\sigma_{2} ; \tau_{2}\right)$ where $\tau_{2}=\left[\tau_{3}-R(q)\right] / d$ and $\sigma_{2}=\sigma_{0}-R(p)-(b / d)\left(\tau_{3}-R(q)\right), \tau_{3}>\max \left[\tau_{0}\right.$, $\left.R(q)+(d / b)\left(\sigma_{0}-R(p)\right)\right]$. Then $\sigma_{2}<0$.

By Theorem 2.10(a), $w(s, t)=\lim _{Y \rightarrow \infty} \lim _{X \rightarrow \infty} w(X, Y ; s, t)$ in $D\left(\sigma_{2} ; \tau_{2}\right)$. For $\tau>\tau_{2}$,

$$
w(X, Y ; 0, t)=\int_{S \cdot R_{X Y}^{0}} e^{-t y} W(x, y) d(x, y)=\int_{0}^{Y} e^{-t y} d y \int_{0}^{y / b} W(x, y) d x
$$

whenever $X>Y / b$. Hence, $\lim _{X \rightarrow \infty} w(X, Y ; 0, t)=\int_{0}^{Y} e^{-t y} U(y) d y$ and $w(0, t)$ $=\int_{0}^{\infty} e^{-t y} U(y) d y$, that is, $f(b t+p, d t+q)=u(t)$. Now $\tau_{2}=\tau_{1}+\epsilon$ where $\epsilon$ is arbitrary.

COROLLARY. Under the hypotheses of the theorem on setting

$$
U_{1}(y)=\frac{1}{d} \int_{0}^{y / b}(-x)^{n} F\left(x, \frac{y-b x}{d}\right) \exp \left(-p x-q\left(\frac{y-b x}{d}\right)\right) d x,
$$

then $L_{t}\left\{U_{1}\right\}$ converges for $\tau>\tau_{1}$ and on setting $f_{t^{n}}=\partial^{n} f / \partial s^{n}$,

$$
u_{1}(t)=f_{s^{n}}(b t+p, d t+q) \text {. }
$$

Proof. By Theorem 2.1(e), $f_{s n}(s, t)=g(s, t)$ where $G(x, y)=(-x)^{n} F(x, y)$ and the bounded convergence holds in $D\left(\sigma_{0} ; \tau_{0}\right)$. Apply the theorem to $G$ instead of $F$ to get $u_{1}(t)=g(b t+p, d t+q)$.

We note that if $L\{F\}$ converges absolutely in Theorem 4.2 and its corollaries, $L\{W\}$ also converges absolutely.

We derive a few other transforms for later reference, assuming that $L\{F\}$ converges boundedly in $D\left(\sigma_{0} ; \tau_{0}\right)$. By Corollary 4 to Theorem 4.2

$$
v_{1}(s, t)=f(s+t, t)
$$

where

$$
\begin{aligned}
& V_{1}(x, y)=F(x, y-x) \\
& V_{1}(x, y)=0,
\end{aligned}
$$$$
\text { in } S: 0 \leqq x \leqq y \text {, }
$$$$
0 \leqq y<x,
$$

and $L\left\{V_{1}\right\}$ converges boundedly for $R(s+t)>\sigma_{0}, R(t)>\tau_{0}$. Setting 


$$
V_{2}(x, y)=\int_{0}^{x} V_{1}(u, y) d u=\int_{0}^{m} F(u, y-u) d u
$$

where $m=\min (x, y)$, it follows from Theorem 2.9 that

$$
v_{2}(s, t)=\frac{f(s+t, t)}{s}
$$

where $L\left\{V_{2}\right\}$ converges $t$-boundedly for $R(s)>0, R(s+t)>\sigma_{0}, R(t)>\tau_{0}$. By Theorem 4.3 , if we set

$$
V_{3}(y)=\int_{0}^{y} F(u, y-u) d u,
$$

then $L_{t}\left\{V_{3}\right\}$ converges for $\tau>\max \left(\sigma_{0}, \tau_{0}\right)$ and

$$
L_{t}\left\{V_{3}\right\}=f(t, t) \text {. }
$$

Hence, for $R(s)>0, R(t)>\max \left(\sigma_{0}, \tau_{0}\right), L\left\{V_{3}\right\}$ converges $t$-boundedly and

$$
v_{3}(s, t)=\frac{f(t, t)}{s} .
$$

A combination of these results yields for $R(s)>0, R(t)>\max \left(\sigma_{0}, \tau_{0}\right)$,

$$
v_{4}(s, t)=\frac{f(s+t, t)-f(t, t)}{s}
$$

where

$$
\begin{array}{ll}
V_{4}(x, y)=-\int_{x}^{v} F(u, y-u) d u, & 0 \leqq x \leqq y, \\
V_{4}(x, y)=0, & 0 \leqq y<x,
\end{array}
$$

and the convergence is $t$-bounded.

5. Convolutions and products of transforms. It is easy to determine the transform of a function $F(x, y)=G(x) \cdot H(y)$ when the one-dimensional transforms $h(s)$ and $g(t)$ exist for $R(s)>\sigma_{0}$, and $R(t)>\tau_{0}$, respectively. If $(s, t)$ $\in D\left(\sigma_{0} ; \tau_{0}\right)$, then

$$
\begin{aligned}
L\{F\} & =\lim _{X, Y \rightarrow \infty} \int_{0}^{X} d x \int_{0}^{Y} e^{-s x-t y} H(x) G(y) d y \\
& =\lim _{X, Y \rightarrow \infty}\left[\int_{0}^{X} e^{-s x} H(x) d x\right]\left[\int_{0}^{Y} e^{-t y} G(y) d y\right]
\end{aligned}
$$

exists and $f(s, t)=h(s) \cdot g(t)$. The convergence is bounded since in one dimension convergence implies bounded convergence.

Consider, for example, the function 


$$
F(x, y)=x^{\alpha} y^{\beta} \quad(\alpha>-1, \beta>-1)
$$

in $Q$. That $\int_{0}^{\infty} e^{-s x} x^{\alpha} d x$ converges absolutely to $\Gamma(\alpha+1) / s^{\alpha+1}$ for $R(s)>0$, $\alpha>-1$ is well known. By the above $L\{F\}$. converges absolutely in $D(0 ; 0)$ and

$$
f(s, t)=\frac{\Gamma(\alpha+1) \Gamma(\beta+1)}{s^{\alpha+1} t^{\beta+1}} .
$$

From Corollary 4 of Theorem 4.2 we have:

THEOREM 5.1. Assume that $a, b, c, d$ are non-negative constants with $\Delta \neq 0$ and that $p$ and $q$ are complex. Given $F(x, y)=H(x) G(y)$, define $W$ as in (4.18). If $L_{\mathbf{0}}\{H\}$ converges for $\sigma>\sigma_{0}, L_{t}\{G\}$ converges for $\tau>\tau_{0}$, then $L\{W\}$ converges boundedly for every $(s, t)$ such that $R(a s+b t+p)>\sigma_{0}, R(c s+d t+q)>\tau_{0}$, and $w(s, t)=h(a s+b t+p) g(c s+d t+q)$.

In particular, if $a>0, b>0$, the function

$$
W(x, y)= \begin{cases}\frac{1}{a} H\left(\frac{x}{a}\right) G\left(y-\frac{b}{a} x\right) & \text { in } y \geqq \frac{b}{a} x \geqq 0, \\ 0 & \text { in } \frac{b}{a} x>y \geqq 0\end{cases}
$$

has the transform

$$
w(s, t)=h(a s+b t) g(t)
$$

provided that $R(a s+b t)>\sigma_{0}, R(t)>\tau_{0}$.

Similarly, for $a>0, b>0$ the function

$$
W(x, y)= \begin{cases}\frac{1}{b} H\left(\frac{y}{b}\right) G\left(x-\frac{a}{b} y\right) & \text { in } \frac{b}{a} x \geqq y \geqq 0, \\ 0 & \text { in } y>\frac{b}{a} x \geqq 0\end{cases}
$$

has the transform

$$
w(s, t)=h(a s+b t) g(s)
$$

provided that $R(s)>\tau_{0}, R(a s+b t)>\sigma_{0}$. Application of Theorem 5.1 to the function $F(x, y)=x^{\alpha} y^{\beta}$ leads to a function $W$ whose transform is

$$
\frac{\Gamma(\alpha+1) \cdot \Gamma(\beta+1)}{(a s+b t+p)^{\alpha+1}(c s+d t+q)^{\beta+1}},
$$

an important function because its denominator is a product of powers of linear functions of $s$ and $t$.

From the above we see that the transform of a product of a function of $x$ 
by a function of $y$ is the product of the transforms. In general, however, the transform of a product of two functions of $x$ and $y$ is not the product of their transforms. However, the latter is the transform of a "convolution function."

The natural extension of the one-dimensional convolution is $F_{1} * F_{2}=C$ where

$$
C(x, y)=\int_{R_{x y}} F_{1}(u, v) F_{2}(x-u, y-v) d(u, v)
$$

and the following theorem concerns its transform(11).

TheOREM 5.2. If $L\left\{F_{1}\right\}$ converges boundedly and $L\left\{F_{2}\right\}$ converges absolutely in $D\left(\sigma_{0} ; \tau_{0}\right)$ or if $L\left\{F_{1}\right\}$ converges s-boundedly and $L\left\{F_{2}\right\}$ converges $t$-boundedly, then $L\left\{F_{1} * F_{2}\right\}$ converges boundedly in $D(c ; d)$ where $c=\max \left(\sigma_{0}, 0\right), d$ $=\max \left(\tau_{0}, 0\right)$, and

$$
L\left\{F_{1 *} F_{2}\right\}=L\left\{F_{1}\right\} \cdot L\left\{F_{2}\right\} .
$$

Proof. We first establish the theorem under the hypothesis: (a) $L\left\{F_{1}\right\}$ and $L\left\{F_{2}\right\}$ converge absolutely at $(s, t)$. Because of the absolute convergence we can write $f_{1} f_{2}$ as a four-fold integral which converges absolutely.

$$
f_{1}(s, t) f_{2}(s, t)=\int_{T} e^{-s\left(x+x^{\prime}\right)-t\left(y+y^{\prime}\right)} F_{1}(x, y) F_{2}\left(x^{\prime}, y^{\prime}\right) d\left(x, y, x^{\prime}, y^{\prime}\right)
$$

where $T: 0 \leqq x, y, x^{\prime}, y^{\prime}<\infty$. Under the linear transformation $x=u, y=v$, $x^{\prime}=w-u, y^{\prime}=z-v$ with determinant +1 the region $T \rightarrow T^{\prime}$ where $T^{\prime}: 0 \leqq w$ $<\infty, 0 \leqq z<\infty, 0 \leqq u \leqq w, 0 \leqq v \leqq z$. Hence,

$$
f_{1}(s, t) f_{2}(s, t)=\int_{T^{\prime}} e^{-s w-t z} F_{1}(u, v) F_{2}(w-u, z-v) d(u, v, w, z)
$$

where this integral converges absolutely and so can be written as a repeated integral in any order. Therefore,

$$
\begin{aligned}
f_{1}(s, t) f_{2}(s, t) & =\int_{Q} e^{-s w-t z}\left[\int_{R_{w z}} F_{1}(u, v) F_{2}(w-u, z-v) d(u, v)\right] d(w, z)=L\{C\}
\end{aligned}
$$

which establishes (5.2) under hypothesis (a).

Now let $L\left\{F_{1}\right\}$ converge boundedly and $L\left\{F_{2}\right\}$ converge absolutely in $D\left(\sigma_{0} ; \tau_{0}\right)$. If we set $\Phi(x, y)=\int_{(0,0)}^{(x, y)} F_{1}(u, v) d(u, v) . L\{\Phi\}$ converges regularly in $D(c ; d)$ and $s t \phi(s, t)=f_{1}(s, t)$. Since $F_{2}$ and $\Phi$ satisfy hypothesis (a), on setting $H=F_{2}{ }^{*} \Phi, L\{H\}$ converges absolutely in $D(c ; d)$ and $h(s, t)=f_{2}(s, t) \phi(s, t)$. But

(i1) Formula 5.2 was established by Amerio under the first hypothesis given in the theorem. using a different proof. P. 729. 


$$
H(x, y)=\int_{0}^{x} d u \int_{0}^{y} F_{2}(u, v) \Phi(x-u, y-v) d v=\int_{(0,0)}^{(x, y)} C(w, z) d(w, z)
$$

where the second equality can be shown by making suitable transformations. Since $L\left\{\left|F_{2}\right|\right\}$ converges to $A$ and $|\Phi(x, y)| \leqq M e^{\sigma x+\tau y}$ in $D(c ; d)$,

$$
|H(x, y)| \leqq M \int_{(0,0)}^{(x, y)}\left|F_{2}(u, v)\right| e^{\sigma(x-u)+\tau(y-v)} d(u, v) \leqq M A e^{\sigma x+\tau y} .
$$

Hence, by Corollary to Theorem 2.9, $L\{C\}$ converges boundedly and $c(s, t)$ $=s t h(s, t)=s t f_{2}(s, t) \phi(s, t)=f_{1}(s, t) f_{2}(s, t)$ in $D(c ; d)$, which establishes the theorem under this hypothesis.

Let $L\left\{F_{1}\right\}$ converge $s$-boundedly and $L\left\{F_{2}\right\}$ converge $t$-boundedly in $D\left(\sigma_{0} ; \tau_{0}\right)$. Then, on setting $\Theta=\int_{0}^{x} F_{1}(u, y) d u$ and $\bar{\Psi}=\int_{0}^{\nu} F_{2}(x, v) d v$, by Theorem $2.8 L\{\bar{\Theta}\}$ and $L\{\bar{\Psi}\}$ converge regularly in $D(c ; d)$ and $f_{1}(s, t)=s \bar{\theta}(s, t), f_{2}(s, t)$ $=t \bar{\psi}(s, t)$ so that $f_{1}(s, t) f_{2}(s, t)=s t \bar{\theta}(s, t) \Psi(s, t)=s t k(s, t)$ where $K=\bar{\Theta} * \bar{\Psi}$ by the first part of the theorem. If $(s, t) \in D(c ; d)$, there exists $\left(\sigma_{2}, \tau_{2}\right)$ with $c<\sigma_{2}<\sigma$, $d<\tau_{2}<\tau$.

$$
\begin{aligned}
|K(x, y)| & \leqq M_{1} M_{2} \int_{(0,0)}^{(x, y)} e^{\sigma_{2} u+\tau_{2} v} e^{\sigma(x-u)+\tau(y-v)} d(u, v) \\
& \leqq M_{1} M_{2} e^{\sigma x+\tau y} /\left[\left(\sigma-\sigma_{2}\right)\left(\tau-\tau_{2}\right)\right]=M e^{\sigma x+\tau y} .
\end{aligned}
$$

One shows that $K(x, y)=\int_{(0,0)}^{(x, y)} C(w, z) d(w, z)$ and, hence, $L\{C\}$ converges boundedly and $c(s, t)=s t k(s, t)=f_{1}(s, t) f_{2}(s, t)$.

If $a>0, b>0$, the function $H(x, y)=\int_{0}^{x / a} Q(u) d u$ for $x / a \leqq y / b$ and $H(x, y)$ $=\int_{0}^{v / b} Q(u) d u$ for $y / b \leqq x / a$ may be conveniently written $H(x, y)=\int_{0}^{m} Q(u) d u$ where $m=\min (x / a, y / b)$. Indeed the same notation can be used for $a \geqq 0$, $b \geqq 0$ if we understand $m=x / a$ when $b=0$ and $m=y / b$ when $a=0$.

Corollary. Let $L\{F\}$ converge boundedly in $D\left(\sigma_{0} ; \tau_{0}\right)$ and $L_{t}\{G\}$ converge absolutely for $\tau>\tau_{1}$. Given $a \geqq 0, b>0$, let $S$ be the set of points: $x \geqq(a / b) y \geqq 0$ and define $W(x, y)=(1 / b) G(y / b)$ in $S, W(x, y)=0$ in $Q-S ;$ let $C=F^{*} W$. Then

$$
C(x, y)=\int_{0}^{m} d \eta \int_{a \eta}^{x} F(\xi-a \eta, y-b \eta) G(\eta) d \xi .
$$

On setting

$$
\sigma_{2}=\max \left(\sigma_{0}, 0, \tau_{1} /\left(a^{2}+b^{2}\right)^{1 / 2}\right), \quad \tau_{2}=\max \left(\tau_{0}, 0, \tau_{1} /\left(a^{2}+b^{2}\right)^{1 / 2}\right),
$$

$L\{C\}$ converges boundedly to $c(s, t)=g(a s+b t) f(s, t) / s$ in $D\left(\sigma_{2} ; \tau_{2}\right)$.

Proof. For $\sigma>0, \tau>\tau_{1}$ the double transform $L\{G\}$ converges absolutely to $g(t) / s$. Then by Corollary 4 to Theorem 4.2 the function $W$ has the double transform $w(s, t)=g(a s+b t) / s$ where the convergence is absolute for all $(s, t)$ such that $\sigma>0, a \sigma+b \tau>\tau_{1}$; in particular, it holds in the region $D\left(\tau_{8} ; \tau_{8}\right)$ 
where $\tau_{3}=\max \left(0, \tau_{1} /\left(a^{2}+b^{2}\right)^{1 / 2}\right)$. Applying Theorem 5.2 to $C=F * W$, we obtain the formula for $c(s, t)$.

Formula (5.3) follows from the definition of the convolution.

Amerio ${ }^{(12)}$ has defined the "convolution about an axis" which we give in our notation. The function $P=G_{a b}^{*} F$ is defined by

$$
P(x, y)=\int_{0}^{m} F(x-a \eta, y-b \eta) G(\eta) d \eta, \quad m=\min \left(\frac{x}{a}, \frac{y}{b}\right),
$$

where $a$ and $b$ are non-negative constants, not both zero.

Theorem 5.3. Let $L\{F\}$ converge boundedly in $D\left(\sigma_{0} ; \tau_{0}\right)$ and let $L_{t}\{G\}$ converge absolutely for $\tau>\tau_{1}$. Then $L\left\{G_{a b}^{*} F\right\}$ converges boundedly to $p(s, t)$ in $D\left(\sigma_{2} ; \tau_{2}\right)$ where $\sigma_{2}$ and $\tau_{2}$ are defined in (5.4) and

$$
p(s, t)=g(a s+b t) f(s, t) .
$$

Proof. Let $b>0, P(x, y)$, and $m$ be defined as in (5.5) and $m^{\prime}$ $=\min (X / a, y / b)$. The set $R_{1}:(0 \leqq x \leqq X ; 0 \leqq \eta \leqq m)$ is the same as the set $R_{2}:\left(0 \leqq \eta \leqq m^{\prime} ; a \eta \leqq x \leqq X\right)$ since a point $(x, \eta)$ satisfying each set of inequalities can be shown to satisfy the other. Therefore, from (5.3)

$$
\begin{aligned}
\int_{0}^{x} P(x, y) d x & =\int_{0}^{x} d x \int_{0}^{m} F(x-a \eta, y-b \eta) G(\eta) d \eta \\
& =\int_{0}^{m^{\prime}} d \eta \int_{a \eta}^{x} F(x-a \eta, y-b \eta) G(\eta) d x=C(X, y) .
\end{aligned}
$$

We have $p(X, Y ; s, t)=\int_{0}^{Y} d y \int_{0}^{X} d x \int_{0}^{m} e^{-s x-t y} F(x-a \eta, y-b \eta) G(\eta) d \eta$. But the set $R_{3}:(0 \leqq x \leqq X ; 0 \leqq y \leqq Y ; 0 \leqq \eta \leqq m)$ can also be written $R_{4}:(0 \leqq \eta$ $\left.\leqq m^{\prime \prime}=\min (X / a, Y / b) ; a \eta \leqq x \leqq X ; b \eta \leqq y \leqq Y\right)$; one proves this by showing that a point $(x, y, \eta)$ satisfying one set of inequalities satisfies the other set also. Therefore,

$$
p(X, Y ; s, t)=\int_{0}^{m^{\prime \prime}} d \eta \int_{a \eta}^{X} d x \int_{b \eta}^{Y} F(x-a \eta, y-b \eta) e^{-s x-t y} G(\eta) d y .
$$

Setting $x^{\prime}=x-a \eta, y^{\prime}=y-b \eta$, we obtain

$$
\begin{aligned}
p(X, Y ; s, t) & =\int_{0}^{m} G(\eta) d \eta \int_{0}^{X-a \eta} d x^{\prime} \int_{0}^{Y-b \eta} e^{-s\left(x^{\prime}+a \eta\right)-t\left(y^{\prime}+b \eta\right)} F\left(x^{\prime}, y^{\prime}\right) d y^{\prime} \\
& =\int_{0}^{m} e^{-(a s+b t) \eta} G(\eta) f(X-a \eta, Y-b \eta ; s, t) d \eta .
\end{aligned}
$$

Let $(s, t) \in D\left(\sigma_{2} ; \tau_{2}\right)$. Since $L\{F\}$ converges boundedly and $a \sigma+b \tau>\tau_{1}$,

(12) Amerio, p. 725. He established Theorem 5.3 using a different proof. 


$$
|p(X, Y ; s, t)| \leqq M \int_{0}^{\infty} e^{-(a \sigma+b \tau) \eta}|G(\eta)| d \eta=M_{1} \text { for all }(X, Y) \text {. }
$$

By Corollary to Theorem 2.9, $L\{P\}$ converges boundedly, $L\{C\}$ converges $t$-boundedly, and $p(s, t)=s c(s, t)$ in $D\left(\sigma_{2} ; \tau_{2}\right)$. By the preceding corollary $p(s, t)=g(a s+b t) f(s, t)$.

When $a>0, b=0$, a parallel proof establishes the result.

When $G(y)=1$, then $g(t)=1 / t$ for $\tau>\tau_{1}=0$ and (5.4) becomes: $\sigma_{2}$ $=\max \left(\sigma_{0}, 0\right)=c, \tau_{2}=\max \left(\tau_{0}, 0\right)=d$. Thus we have:

Corollary. If $L\{F\}$ converges boundedly in $D\left(\sigma_{0}, \tau_{0}\right)$, then for $a$ and $b$ non-negative and not both zero, set $V(x, y)=\int_{0}^{m} F(x-a \eta, y-b \eta) d \eta, m$ $=\min (x / a, y / b)$. Then $L\{V\}$ converges boundedly for $(s, t) \in D(c ; d)$ and $v(s, t)$ $=f(s, t) /(a s+b t)$.

As an immediate consequence of Theorem 5.2 and the example considered at the beginning of this section, we have the following theorem:

TheOREM 5.4. Let $L\{F\}$ converge boundedly at $\left(s_{0}, t_{0}\right)$ and set $c=\max \left(\sigma_{0}, 0\right)$, $d=\max \left(\tau_{0}, 0\right)$. Then for all $(s, t) \in D(c ; d), L\left\{\Phi_{a \beta}\right\}$ converges boundedly and

$$
\phi_{\alpha \beta}(s, t)=\frac{f(s, t)}{s^{\alpha+1} t^{\beta+1}}, \quad \alpha>-1, \beta>-1,
$$

where

$$
\Phi_{\alpha \beta}(x, y)=\frac{1}{\Gamma(\alpha+1) \Gamma(\beta+1)} \int_{R_{x y}} F(u, v)(x-u)^{\alpha}(y-v)^{\beta} d(u, v) .
$$

6. Transforms of difference quotient type. There is no function $F(x, y)$ such that $L\{F\}$ converges boundedly at a finite point $\left(s_{0}, t_{0}\right)$ to $1 /(s-t)$. For suppose there were such a function and assume further that $R\left(t_{0}\right) \geqq R\left(s_{0}\right)$. The point $\left(t_{0}+1, t_{0}+1\right)$ lies in the region of bounded convergence since $R\left(t_{0}+1\right)>R\left(t_{0}\right) \geqq R\left(s_{0}\right)$ and $R\left(t_{0}+1\right)>R\left(t_{0}\right)$. However, $1 /(s-t)$ is not even defined at this point.

Certain functions of $s$ and $t$, when combined properly with $1 /(s-t)$, do result in transforms as we shall show. The corresponding functions of $x$ and $y$ are integrals of a type important in partial differential equations.

Theorem 6.1. Let $L\{F\}$ converge boundedly at $\left(s_{0}, t_{0}\right)$ and set $\bar{\sigma}$ $=\max \left(\sigma_{0}, \tau_{0}\right)$. For $(s, t) \in D(\bar{\sigma} ; \bar{\sigma})$ define

$$
\begin{array}{ll}
\gamma(s, t)=\frac{f(s, t)-f(t, t)}{s-t}, & s \neq t, \\
\gamma(s, t)=f_{\mathrm{s}}(t, t), & s=t,
\end{array}
$$




$$
\begin{array}{rlrl}
\lambda(s, t) & =\frac{f(s, t)-f(s, s),}{t-s}, & t \neq s, \\
\lambda(s, t)=f_{t}(s, s), & t=s .
\end{array}
$$

Then $\gamma(s, t)=L\{\Gamma\}$ and $\lambda(s, t)=L\{\Lambda\}$ converge boundedly in $D(\bar{\sigma} ; \bar{\sigma})$ where

$$
\begin{aligned}
& \Gamma(x, y)=-\int_{0}^{y} F(x+z, y-z) d z, \\
& \Lambda(x, y)=-\int_{0}^{x} F(x-z, y+z) d z .
\end{aligned}
$$

Proof. Let $V_{4}(x, y)$ be defined as in (4.26). Then $L\left\{V_{4}\right\}$ converges boundedly and its transform is given by $(4.25)$ in $R(s)>0, R(t)>\max \left(\sigma_{0}, \tau_{0}\right)$. By Corollary 4 to Theorem 4.2 , since $V_{4}(x, y)=0$ in $0 \leqq y<x$, the function $W$ defined by $W(x, y)=V_{4}(x, y+x)=-\int_{x}^{y+x} F(u, y+x-u) d u=\Gamma(x, y)$ has the double transform $v_{4}(s-t, t)=[f(s, t)-f(t, t)] /(s-t)=\gamma(s, t)$ where the convergence is bounded for $R(s-t)>0, R(t)>\max \left(\sigma_{0}, \tau_{0}\right)$, that is, for $R(s)$ $>R(t)>\max \left(\sigma_{0}, \tau_{0}\right)$.

In an analogous fashion one shows that $M(x, y)=0$ for $y \geqq x \geqq 0, M(x, y)$ $=\int_{0}^{v} F(x-v, v) d v$ for $x>y \geqq 0$ has the double transform $m(s, t)=[f(s, s+t)$ $-f(s+t, s+t)] / t$ in the region $R(s)>\max \left(\sigma_{0}, \tau_{0}\right), R(t)>0$. Therefore, $\gamma(s, t)=-m(s, t-s)=L\{\Gamma\}$ for $R(t)>R(s)>\max \left(\sigma_{0}, \tau_{0}\right)$.

Consider any $(s, t) \in D(\bar{\sigma} ; \bar{\sigma})$. There is a point $\left(s_{1}, t_{1}\right)$ such that $\bar{\sigma}<\sigma_{1}<\sigma$, $\bar{\sigma}<\tau_{1}<\tau$, and $\sigma_{1}>\tau_{1}$. By the first part of the proof $L\{\Gamma\}$ converges boundedly at $\left(s_{1}, t_{1}\right)$ and, hence, by Theorem 2.1 , at $(s, t)$. Thus $L\{\Gamma\}=\bar{\gamma}(s, t)$ exists in $D(\bar{\sigma} ; \bar{\sigma})$ and by the same theorem is an analytic function there. We know that $\gamma(s, t)=\bar{\gamma}(s, t)$ when $R(s) \neq R(t)$. The analyticity of $\bar{\gamma}$ insures that the formula holds also for $R(s)=R(t), s \neq t$. Finally, for $s=t, \bar{\gamma}(t, t)=\lim _{s \rightarrow t} \bar{\gamma}(s, t)$ $=\lim _{s \rightarrow t} \gamma(s, t)=f_{s}(t, t)$.

The second part can be obtained from the first by proper interchange of variables.

Corollary. Let $L\{F\}$ converge boundedly in $D\left(\sigma_{0} ; \tau_{0}\right)$. Define for $m>0, p$ complex, and $(s, t) \in D\left(\sigma_{0}, \tau\right)$

$$
\begin{array}{ll}
\gamma_{1}(s, t)=\frac{f(s, t)-f(m t+p, t)}{s-m t-p}, & s \neq m t+p, \\
\gamma_{1}(s, t)=f_{s}(m t+p, t), & s=m t+p,
\end{array}
$$

and for $(s, t) \in D\left(\bar{\sigma} ; \tau_{0}\right)$

$$
\begin{array}{ll}
\lambda_{1}(s, t)=\frac{f(s, t)-f(s, m s+p)}{t-m s-p}, & t \neq m s+p, \\
\lambda_{1}(s, t)=f_{t}(s, m s+p), & t=m s+p .
\end{array}
$$


where $\bar{\sigma}=\max \left(\sigma_{0},\left[\tau_{0}-\mathcal{R}(p)\right] / m\right), \bar{\tau}=\max \left(\tau_{0},\left[\sigma_{0}-\mathcal{R}(p)\right] / m\right)$. If

$$
\begin{aligned}
& \Gamma_{1}(x, y)=-\int_{0}^{y / m} e^{-p z} F(x+z, y-m z) d z, \\
& \Lambda_{1}(x, y)=-\int_{0}^{x / m} e^{-p z} F(x-m z, y+z) d z,
\end{aligned}
$$

then $L\left\{\Gamma_{1}\right\}=\gamma_{1}(s, t)$ and $L\left\{\Lambda_{1}\right\}=\lambda_{1}(s, t)$ where the convergence is bounded in $D(m \bar{\tau}+R(p) ; \bar{\tau})$ and $D(\bar{\sigma} ; m \bar{\sigma}+R(p))$ respectively.

Proof. Set $\pi(s, t)=f(s,(t-p) / m)$. For $R(s)>\sigma_{0}, R(t)>m \tau_{0}+R(p), \pi(s, t)$ $=L\left\{F_{1}\right\}$ where $F_{1}(x, y)=m F(x, m y) e^{p y}$ by Corollaries 2 and 3 to Theorem 4.2. Setting $\sigma_{2}=\max \left(\sigma_{0}, m \tau_{0}+R(p)\right)$ and

$$
\begin{array}{rlrl}
c(s, t) & =\frac{\pi(s, t)-\pi(t, t)}{s-t}, & s \neq t, \\
c(s, t)=\pi_{s}(t, t), & s=t,
\end{array}
$$

we find that $L\{C\}=c(s, t)$ in $D\left(\sigma_{2} ; \sigma_{2}\right)$ where $C(x, y)=-\int_{0}^{\nu} F_{1}(x+z, y-z) d z$. By definition $c(s, m t+p)$ is given by (6.5) and the complex linear transformation yields $\gamma_{1}(s, t)=L\left\{\Gamma_{1}\right\}$.

To prove the second part of the corollary, use the first result to obtain the function whose transform is $\gamma_{1}(t, s)$, interchanging the first and second variables in $F$.

THEOREM 6.2. Let $L\{F\}$ converge boundedly in $D\left(\sigma_{0} ; \tau_{0}\right)$ and define a function $\gamma_{n}$ for $m>0$, $p$ complex, $n$ a positive integer, and $(s, t) \in D\left(\sigma_{0} ; \bar{\tau}\right)$ by the formulas

$$
\begin{array}{ll}
\gamma_{n}(s, t)=\frac{f(s, t)-\sum_{i=0}^{n-1} \frac{1}{i !} f_{s^{i}}(m t+p, t)(s-m t-p)^{i}}{(s-m t-p)^{n}}, & s \neq m t+p, \\
\gamma_{n}(s, t)=\frac{1}{n !} f_{s^{n}}(m t+p, t), & s=m t+p,
\end{array}
$$

where $\bar{\tau}=\max \left(\tau_{0},\left[\sigma_{0}-R(p)\right] / m\right)$.

Then in $D\left(\sigma_{0} ; \bar{\tau}\right)$

$$
\begin{array}{ll}
\gamma_{n}(s, t)=\frac{\gamma_{n-1}(s, t)-\gamma_{n-1}(m t+p, t)}{s-m t-p}, & s \neq m t+p, \\
\gamma_{n}(s, t)=\left.\frac{\partial \gamma_{n-1}}{\partial s}\right|_{s=m t+p}, & s=m t+p,
\end{array}
$$

on setting $\gamma_{0}(s, t)=f(s, t)$. Furthermore, in $D(m \bar{\tau}+R(p) ; \bar{\tau}) L\left\{\Gamma_{n}\right\}$ converges 
boundedly to $\gamma_{n}(s, t)$ where

$$
\Gamma_{n}(x, y)=\frac{(-1)^{n}}{(n-1) !} \int_{0}^{y / m} e^{-p z} F(x+z, y-m z) z^{n-1} d z .
$$

Proof. Since $D\left(\sigma_{0} ; \bar{\tau}\right) \subseteq D\left(\sigma_{0} ; \tau_{0}\right), f(s, t)$ is analytic and so has partial derivatives of all orders in $D\left(\sigma_{0} ; \bar{\tau}\right)$. Hence, the functions defined by (6.9) all exist. For $n=1,(6.10)$ follows from the definitions of $\gamma_{1}$ and $\gamma_{0}$.

If we substitute the Taylor's expansion of $f(s, t)$ about $s=m t+p$ in the numerator of (6.9), we see that for $s \neq m t+p$

$$
\gamma_{n}(s, t)=\sum_{i=n}^{\infty} \frac{1}{i !} f_{g^{i}}(m t+p, t)(s-m t-p)^{i-n} \quad(n=1,2,3, \cdots) .
$$

We note that this formula also holds for $s=m t+p$, from the second part of (6.9). Furthermore, since the limit of the right-hand side, as $s \rightarrow m t+p$, exists and equals $f_{s^{n}}(m t+p, t) / n !, \lim _{s \rightarrow m t+p} \gamma_{n}(s, t)$ exists and equals $\gamma_{n}(m t+p, t)$.

Suppose $n \geqq 2$ and $s \neq m t+p$. Then from (6.11a)

$$
\begin{aligned}
\frac{\gamma_{n-1}(s, t)}{}-\gamma_{n-1}(m t+p, t) \\
s-m t-p \\
=\frac{\sum_{i=n-1}^{\infty} \frac{1}{i !} f_{s^{i}}(m t+p, t)(s-m t-p)^{i-n+1}-\frac{1}{(n-1) !} f_{s^{n-1}}(m t+p, t)}{s-m t-p} \\
=\sum_{i=n}^{\infty} \frac{1}{i !} f_{s^{i}}(m t+p, t)(s-m t-p)^{i-n}=\gamma_{n}(s, t)
\end{aligned}
$$

which is the first part of (6.10). On taking the limit as $s \rightarrow m t+p$, it follows that $\partial \gamma_{n-1} /\left.\partial s\right|_{s-m t+p}$ exists and equals $\gamma_{n}(m t+p, t)$ which is the second part of (6.10).

By the previous corollary, for $n=1, L\left\{\Gamma_{n}\right\}$ converges boundedly to $\gamma_{n}(s, t)$ in $D(m \bar{\tau}+R(p) ; \bar{\tau})$. Assume that this statement holds for $n=k$. Using $\gamma_{k}(s, t)$ as $f(s, t)$ of the previous corollary, we find that $\gamma_{k+1}(s, t)=L\{V\}$ in $D(m \bar{\tau}+R(p) ; \bar{i})$ where

$$
V(x, y)=-\int_{0}^{y / m} e^{-p \xi} \Gamma_{k}(x+\xi, y-m \xi) d \xi .
$$

Substitution for $\Gamma_{k}$ yields

$$
\begin{aligned}
V(x, y) & =-\int_{0}^{y / m} e^{-p \xi}\left[\frac{(-1)^{k}}{(k-1) !} \int_{0}^{(y-m \xi) / m} e^{-p z} F(x+\xi+z, y-m \xi-m z) z^{k-1} d z\right] d \xi \\
& =\frac{(-1)^{k+1}}{(k-1) !} \int_{S_{y}} e^{-p(\xi+z)} F(x+\xi+z, y-m \xi-m z) z^{k-1} d(\xi, z)
\end{aligned}
$$


where $S_{y}: 0 \leqq \xi \leqq y / m, 0 \leqq z \leqq(y-m \xi) / m$. On making the transformation $\xi=\eta-w, z=w$, we see that $V(x, y)=\Gamma_{k+1}(x, y)$, the last equality resulting from performing the integration with respect to $w$. Hence, $L\left\{\Gamma_{n}\right\}$ converges boundedly to $\gamma_{n}$ for $n=k+1$ and by induction for all $n$.

We now apply the last two theorems to $F(x, y)=H(x)$.

TheOREM 6.3. Let $L_{s}\{H\}$ converge for $R(s)>\sigma_{1}$ and define in $D\left(\sigma_{1} ; \sigma_{1}\right)$

$$
\begin{array}{llrl}
\omega(s, t) & =\frac{h(s)-h(t)}{s-t}, & & s \neq t, \\
\omega(s, t) & =h^{\prime}(t), & s & =t .
\end{array}
$$

Then $\omega(s, t)=L\{\Omega\}$ where

$$
\Omega(x, y)=-H(x+y)
$$

and the convergence is bounded in $D(\bar{\sigma} ; \bar{\sigma})$ where $\bar{\sigma}=\max \left(\sigma_{1}, 0\right)$.

Proof. Let $F(x, y)=H(x)$; then $L\{F\}$ converges to $h(s) / t$ in $D\left(\sigma_{1} ; 0\right)$. If $\bar{\sigma}=\max \left(\sigma_{1}, 0\right)$, define in $D(\bar{\sigma} ; \bar{\sigma})$

$$
\begin{array}{ll}
\gamma(s, t)=\frac{f(s, t)-f(t, t)}{s-t}=\frac{h(s)-h(t)}{t(s-t)}, & s \neq t, \\
\gamma(s, t)=f_{8}(t, t)=h^{\prime}(t) / t, & s=t .
\end{array}
$$

By Theorem 6.1, $\gamma(s, t)$ is the double transform of

$$
\Gamma(x, y)=-\int_{0}^{y} F(x+z, y-z) d z=-\int_{0}^{y} H(x+z) d z
$$

in $D(\bar{\sigma} ; \bar{\sigma})$ and by definition of $\omega, \gamma(s, t)=\omega(s, t) / t$.

Let $G(x, y)=-H(x+y)$ and $\Gamma(x, y)=\int_{0}^{y} G(x, z) d z$. Since $L_{s}\{H\}$ converges for $\sigma>\sigma_{1},\left|\int_{0}^{x} H(u) d u\right| \leqq M e^{\sigma x}$ for $\sigma>\bar{\sigma}$. Therefore, $|\Gamma(x, y)|=\left|\int_{x}^{x+y} H(u) d u\right|$ $\leqq M\left[e^{\sigma(x+y)}+e^{\sigma x}\right] \leqq 2 M e^{\sigma x+\sigma y}$ for $\sigma>\bar{\sigma}$. Hence, by Corollary 1 to Theorem 2.8, $L\{G\}$ converges $t$-boundedly and $g(s, t)=t \gamma(s, t)=\omega(s, t)$ in $D(\bar{\sigma} ; \bar{\sigma})$.

THEOREM 6.4. Let $L_{s}\{H\}$ converge to $h(s)$ for $R(s)>\sigma_{1}$, and define a function $\omega_{n}$ for $m>0, p$ complex, $n$ a positive integer and $(s, t) \in D\left(\sigma_{1} ; \bar{\tau}\right)$ by the formulas

$$
\begin{array}{ll}
\omega_{n}(s, t)=\frac{h(s)-\sum_{i=0}^{n-1} \frac{1}{i !} h^{(i)}(m t+p)(s-m t-p)^{i}}{(s-m t-p)^{n}}, & s \neq m t+p, \\
\omega_{n}(s, t)=\frac{1}{n !} h^{(n)}(m t+p), & s=m t+p,
\end{array}
$$

where $\bar{\tau}=\max \left(0,\left[\sigma_{1}-R(p)\right] / m\right), \bar{\sigma}=\max \left(0, \sigma_{1}\right)$. 
Then in $D\left(\sigma_{1} ; \bar{\tau}\right)$

$$
\begin{array}{ll}
\omega_{n}(s, t)=\frac{\omega_{n-1}(s, t)-\omega_{n-1}(m t+p, t)}{s-m t-p}, & s \neq m t+p, \\
\omega_{n}(s, t)=\left.\frac{\partial \omega_{n-1}}{\partial s}\right|_{s=m t+p}, & s=m t+p,
\end{array}
$$

where $\omega_{0}(s, t)=h(s)$ and $n=1,2,3, \cdots$. Furthermore, in

$$
D(\bar{\sigma} ; \max (0,[\bar{\sigma}-R(p)] / m))
$$

$L\left\{\Omega_{n}\right\}$ converges boundedly to $\omega_{n}(s, t)$, where

$$
\Omega_{n}(x, y)=\frac{(-1)^{n} y^{n-1}}{m^{n}(n-1) !} H\left(x+\frac{y}{m}\right) e^{-p y / m} .
$$

If $\omega_{n}$ is defined as in (6.14)

$$
\omega_{n}(s, t)=\left.\frac{1}{(n-1) !} \frac{\partial^{n-1} \omega}{\partial t^{n-1}}\right|_{s=m t+p} .
$$

Proof. Let $F(x, y)=H(x)$. Then $L\{F\}$ converges to $f(s, t)=h(s) / t$ in $D\left(\sigma_{1} ; 0\right)$. Defining $\gamma_{n}(s, t)$ as in (6.9), we see that

$$
\gamma_{n}(s, t)=\omega_{n}(s, t) / t
$$

for $R(s)>\sigma_{1}, R(t)>\bar{\tau}$, and $n=0,1,2, \ldots$. By Theorem 6.2 formulas (6.10) hold in $D\left(\sigma_{1} ; \bar{\tau}\right)$; substituting from (6.17) one obtains (6.15). Furthermore,

$$
\Gamma_{n}(x, y)=\frac{(-1)^{n}}{(n-1) !} \int_{0}^{y / m} e^{-p z} H(x+z) z^{n-1} d z
$$

and $L\left\{\Gamma_{n}\right\}$ converges boundedly to $\omega_{n}(s, t) / t$ in $D(m \tau+R(p) ; \tau)$.

But $\Gamma_{n}(x, y)=\int_{0}^{v} \Omega_{n}(x, z) d z$ where $\Omega_{n}$ is defined as in (6.16). By Theorem 6.3, $\omega(s, t)=L\{\Omega\}$ in $D(\bar{\sigma} ; \bar{\sigma})$ where $\Omega(x, y)=-H(x+y)$. By Corollary 4 to Theorem 4.2, $\omega_{1}(s, t)=\omega(s, m t+p)=L\left\{\Omega_{1}\right\}$ in $D(\bar{\sigma} ;[\bar{\sigma}-R(p)] / m)$ where $\Omega_{1}(x, y)=-(1 / m) H(x+y / m) e^{-p y / m}$ in $Q$.

By Theorem 2.1(e), $\quad \partial^{n-1} \omega_{1}(s, t) / \partial t^{n-1}=L\{P\} \quad$ where $P(x, y)$ $=\left((-1)^{n} / m\right) H(x+y / m) y^{n-1} e^{-p y / m}+m^{n-1}(n-1) ! \Omega_{n}(x, y)$. Since $\partial^{n-1} \omega_{1}(s, t) / \partial t^{n-1}$ $=m^{n-1} \omega_{t^{n-1}}(s, m t+p)$, it follows that $L\left\{\Omega_{n}\right\}$ converges boundedly to $(1 /(n-1) !) \omega_{t^{n-1}}(s, m t+p)=k(s, t)$ in $D(\bar{\sigma} ;[\bar{\sigma}-R(p)] / m)$. Hence, $L\left\{\Gamma_{n}\right\}$ converges $s$-boundedly in $D(\bar{\sigma} ; \max (0,[\bar{\sigma}-R(p)] / m))$ and

$$
k(s, t)=t \gamma_{n}(s, t)=\omega_{n}(s, t) .
$$

7. Partial fractions expansions. Partial fractions play an important role in the solution by transforms of ordinary differential equations, but they do not enjoy a parallel significance in partial differential equations where the 
prescribed values are arbitrary functions. We present two theorems indicating the type of result obtainable.

Theorem 7.1. Let $f(s, t)=p(s, t) / q(s, t)$ where $p(s, t)$ and $q(s, t)$ are polynomials in $s$ and $t$ and $q(s, t)$ has the form

$$
q(s, t)=\left(s+a_{1} t\right)\left(s+a_{2} t\right) \cdots\left(s+a_{n} t\right),
$$

the $a_{i}$ 's being positive and distinct. Hence, $f$ is defined everywhere except along the sets $s+a_{i} t=0$. Assume further that $p(s, t)$ does not have any $\left(s+a_{i} t\right)$ as a factor and that the degree of $p(s, t)$ in $s$ and $t$ is at least two less than that of $q(s, t)$. Set

$$
\begin{array}{ll}
p(s, t)=\sum_{i, j=0, i+j \leqq n-2}^{n-2} \alpha_{i j} s^{i} t^{i}, & \\
\Gamma_{m}(k)=\sum_{p=0}^{m} \alpha_{p, m-p}\left(-a_{k}\right)^{p} & (0 \leqq m \leqq n-2),
\end{array}
$$

and

$$
\bar{q}(s)=q(s, 1)=\left(s+a_{1}\right)\left(s+a_{2}\right) \cdots\left(s+a_{n}\right) .
$$

Then $f(s, t)=L\{F\}$ in $D(0 ; 0)$ where

$$
F(x, y)=\sum_{k=1}^{n}\left[\frac{1}{\bar{q}^{\prime}\left(-a_{k}\right)} \sum_{r=0}^{n-2} \Gamma_{n-r-2}(k) \frac{\left\{y-a_{k} x\right\} r}{r !}\right]
$$

and $\left\{y-a_{k} x\right\}$ has the interpretation $\left\{y-a_{k} x\right\}=0$ when $y<a_{k} x$ and $\left\{y-a_{k} x\right\}$ $=y-a_{k} x$ when $y>a_{k} x$.

Proof. Holding $t$ fixed and expanding $f$ by partial fractions, we can write for $s+a_{h} t \neq 0$

$$
f(s, t)=\sum_{h=1}^{n} \frac{C_{h}(t)}{s+a_{h} t}
$$

where $C_{k}(t)=p\left(-a_{k} t, t\right) / q_{s}\left(-a_{k} t, t\right)$. From (7.1) we see that

$$
q_{s}\left(-a_{k} t, t\right)=\prod_{i=1, i \neq k}^{n}\left(a_{i}-a_{k}\right) t^{n-1}=\bar{q}^{\prime}\left(-a_{k}\right) t^{n-1}
$$

and from (7.2) that

$$
\begin{aligned}
p\left(-a_{k} t, t\right) & =\sum_{i, j=0, i+j \leqq n-2}^{n-2} \alpha_{i j}\left(-a_{k} t\right)^{i} t^{i}=\sum_{i, j=0, i+j \leqq n-2}^{n-2} \alpha_{i j}\left(-a_{k}\right)^{i t^{i+}} \\
& =\sum_{m=0}^{n-2} \Gamma_{m}(k) t^{m} .
\end{aligned}
$$


Thus

$$
f(s, t)=\sum_{h=1}^{n} \frac{\sum_{m=0}^{n-2} \Gamma_{m}(h) t^{m}}{\bar{q}^{\prime}\left(-a_{h}\right) t^{n-1}\left(s+a_{h} t\right)} \quad \text { for } s \neq-a_{h} t
$$

and in particular this holds for all $(s, t)$ in $D(0 ; 0)$. If $\mu$ is a positive integer, $1 / t^{\mu}\left(s+a_{k} t\right)$ is the transform of $\left\{y-a_{k} x\right\}^{\mu-1} /(\mu-1)$ ! for $(s, t) \in D(0 ; 0)$. Thus we find that $f(s, t)=L\{F\}$ in $D(0 ; 0)$ where $F$ is defined by (7.3). This function takes a step at each of the $n$ rays $y=a_{k} x$.

We proceed to show the effect of a repeated linear factor.

Theorem 7.2. Let $f(s, t)=p(s, t) / q(s, t)$ where $p(s, t)$ and $q(s, t)$ are polynomials in $s$ and $t$ and $q(s, t)$ has the form

$$
q(s, t)=(s+a t)^{r}\left(s+a_{1} t\right)\left(s+a_{2} t\right) \cdots\left(s+a_{n} t\right) \quad(r>1 ; n \geqq 2)
$$

all the $a$ 's being positive and distinct. Assume further that $p(s, t)$ does not have $s+a_{i}$ tor $s+a t$ as a factor and that the degree of $p(s, t)$ is at most $n-2$. Set

$$
p(s, t)=\sum_{i, j=0, i+j \leqq n-2}^{n-2} \alpha_{i j} s^{i} t^{i}
$$

and define $\bar{q}(s)$ and $\Gamma_{m}(k)$ as in (7.2). Then

$$
f(s, t)=L\{F\}
$$

in $D(0 ; 0)$ where

$$
\begin{aligned}
F(x, y)=\sum_{h=1}^{n} \sum_{m=0}^{n-2} \frac{\Gamma_{n-m-2}(h)}{\bar{q}^{\prime}\left(-a_{h}\right)} & {\left[\frac{\left\{y-a_{h} x\right\}^{m+r}}{(m+r) !\left(a-a_{h}\right)^{r}}\right.} \\
& \left.-\sum_{k=1}^{n} \frac{\{y-a x\}^{m+k} x^{r-k}}{(m+k) !(r-k) !\left(a-a_{h}\right)^{k-1}}\right] .
\end{aligned}
$$

Proof. Let $\phi(s, t)=(s+a t) r f(s, t)=p(s, t) /\left(s+a_{1} t\right) \cdots\left(s+a_{n} t\right)$. Since $\phi$ is just the $f$ of Theorem 7.1, formula (7.4) holds with $\phi$ instead of $f$. From this one obtains, on differentiating $k$ times with respect to $s(0 \leqq k \leqq r-1)$ and then substituting $s=-a t$,

$$
A_{k}(t) \equiv \frac{1}{k !} \phi_{s^{k}}(-a t, t)=-\sum_{h=1}^{n} \sum_{m=0}^{n-2} \frac{\Gamma_{m}(h) t^{m-n-k}}{\bar{q}^{\prime}\left(-a_{h}\right)\left(a-a_{h}\right)^{k}} .
$$

As in the previous theorem

$$
C_{h}(t) \equiv p\left(-a_{h} t, t\right) / q_{s}\left(-a_{h} t, t\right)=\frac{\sum_{m=0}^{n-2} \Gamma_{m}(h) t^{m}}{\left(a-a_{h}\right)^{r} \bar{q}^{\prime}\left(-a_{h}\right) t^{n+r-1}} .
$$


Expansion in partial fractions holding $t$ fixed yields

$$
f(s, t)=\sum_{h=1}^{n} \frac{C_{h}(t)}{s+a_{h} t}+\sum_{k=0}^{r-1} \frac{A_{h}(t)}{(s+a t)^{r-k}} .
$$

Substituting from the above and using the fact that for a positive integer $\mu, 1 / t^{\mu}(s+a t)^{h}$ is the transform of $x^{h-1}\{y-a x\}^{\mu-1} /(h-1) !(\mu-1)$ ! in $D(0 ; 0)$, we obtain (7.5).

TAYLOR INSTRUMENT COMPANIES, ROCHESTER, N. Y.

UNIVERSITY OF ROCHESTER,

ROCHESTER, N. Y. 